ARTICLE

\title{
Butyrophilin-like proteins display combinatorial diversity in selecting and maintaining signature intraepithelial $\gamma \delta$ T cell compartments
}

\author{
Anett Jandke (10 1, Daisy Melandri (10 1,2, Leticia Monin (10 1, Dmitry S. Ushakov (1) 1,2, Adam G. Laing 1,2, \\ Pierre Vantourout (10 ${ }^{1,2}$, Philip East ${ }^{3}$, Takeshi Nitta (1) ${ }^{4}$, Tomoya Narita ${ }^{5}$, Hiroshi Takayanagi ${ }^{4}$, \\ Regina Feederle (i) ${ }^{6} \&$ Adrian Hayday (1) ${ }^{1,2}{ }^{凶}$
}

Butyrophilin-like (Btnl) genes are emerging as major epithelial determinants of tissueassociated $\gamma \delta \mathrm{T}$ cell compartments. Thus, the development of signature, murine TCR $\gamma \delta^{+}$ intraepithelial lymphocytes (IEL) in gut and skin depends on Btnl family members, Btnl1 and Skint1, respectively. In seeking mechanisms underlying these profound effects, we now show that normal gut and skin $\gamma \delta$ IEL development additionally requires Btnl6 and Skint2, respectively, and furthermore that different Btnl heteromers can seemingly shape different intestinal $\gamma \delta^{+}$IEL repertoires. This formal genetic evidence for the importance of Btnl heteromers also applied to the steady-state, since sustained Btnl expression is required to maintain the signature TCR.V $\gamma 7^{+}$IEL phenotype, including specific responsiveness to Btnl proteins. In sum, Btnl proteins are required to select and to maintain the phenotypes of tissue-protective $\gamma \delta$ IEL compartments, with combinatorially diverse heteromers having differential impacts on different IEL subsets.

\footnotetext{
${ }^{1}$ Immunosurveillance Laboratory, The Francis Crick Institute, 1 Midland Road, London NW11AT, UK. ${ }^{2}$ Peter Gorer Department of Immunobiology, School of Immunology and Microbial Sciences, King's College London, Great Maze Pond, London Bridge, London SE19RT, UK. ${ }^{3}$ Bioinformatics and Biostatistics Laboratory, The Francis Crick Institute, 1 Midland Road, London NW11AT, UK. ${ }^{4}$ Department of Immunology, Graduate School of Medicine and Faculty of Medicine, University of Tokyo, Hongo 7-3-1, Bunkyo-ku, Tokyo 113-0033, Japan. ${ }^{5}$ Department of Pharmacotherapy, Research Institute of Pharmaceutical Sciences, Musashino University, Nishitokyo, Tokyo 202-8585, Japan. ${ }^{6}$ Monoclonal Antibody Core Facility, Institute for Diabetes and Obesity, Helmholtz Zentrum, München, German Research Centre for Environmental Health, 85764 Neuherberg, Germany. ${ }^{\circledR}$ email: Adrian.hayday@crick.ac.uk
} 
$\mathrm{F}$ rom jawless vertebrates through to humans, many extralymphoid tissues harbour distinct immune cell populations. Those include tissue-resident memory $\left(T_{\mathrm{RM}}\right)$ cells that infiltrate tissues after antigen priming in lymphoid tissues, and remain well-placed to respond to local antigen recurrence ${ }^{1}$. In addition, various myeloid and lymphoid cells, including macrophages $^{2}$, T-regulatory cells ${ }^{3}$ and $\gamma \delta \mathrm{T}$ cells become associated with tissues developmentally, remaining in situ lifelong ${ }^{4-8}$. Such cells are implicated in protecting tissue integrity, and $\gamma \delta \mathrm{T}$-cell deficiency is causally linked to cancer, tissue inflammation and defective wound healing ${ }^{9-12}$.

In addition, the molecular phenotypes of local T cells commonly emphasise their relationships to specific anatomical sites $^{3}$. Thus, murine $\gamma \delta \mathrm{T}$ cells, which were revealed some 30 years ago to be prototypic tissue-associated $\mathrm{T}$ cells, display tissue-restricted $\mathrm{T}$-cell receptor (TCR) repertoires, including $\mathrm{V} \gamma 5 \mathrm{~V} \delta 1$ in the epidermis, $\mathrm{V} \gamma 6 \mathrm{~V} \delta 1$ in the uterus, dermis and lung, and $\mathrm{V} \gamma 7^{+}$cells expressing a variety of $\mathrm{V} \delta$ chains in the small intestine ${ }^{13}$. Nonetheless, how such TCRs contributed to tissue protection remained enigmatic, particularly given that $\mathrm{V} \gamma 5 \mathrm{~V} \delta 1^{+}$dendritic epidermal T cells (DETC) were shown to use innate receptors, specifically NKG2D, to respond rapidly to epithelial cell dysregulation ${ }^{14,15}$.

Recently however, signature TCRs were shown to mediate the tissue-specific selection of $\gamma \delta$ T cells by members of the heretofore enigmatic butyrophilin-like (Btnl) subfamily of B7 genes. Thus, Btnl1 ${ }^{-/-}$mice mostly lack intestinal $\mathrm{V} 7^{+}$cells ${ }^{16}$, while mice deficient in Skint1 (a Btnl-related gene) specifically lack V $\gamma 5 \mathrm{~V} \delta 1^{+}$ DETC $^{17,18}$. The conservation of this biology became evident when human colonic $\mathrm{V} \gamma 4^{+}$cells were shown to be specifically regulated by BTNL3 ${ }^{16,19}$, while Butyrophilin $3 \mathrm{~A} 1$ (BTN3A1) and $\mathrm{BTN} 2 \mathrm{~A} 1^{20}$ were found to be critical for signature responses of human peripheral blood $\mathrm{V} \gamma 9 \mathrm{~V} \delta 2^{+}$cells to low molecular mass phosphoantigens such as isopentenyl pyrophosphate ${ }^{21-23}$. Moreover, dysregulation of the BTNL3-V $\gamma 4^{+}$axis has been implicated in celiac disease ${ }^{24}$. Thus, there is considerable interest in the mechanisms by which Skint/Btnl/BTN genes exert their effects.

Consistent with their regulation of $\gamma \delta$ T-cell subsets defined by their TCRs, Btnl/BTNL proteins have emerged as bona fide T-cell selecting ligands akin to MHC or CD1. In addition, evidence from cell culture and biochemical experiments argues that Btnl/BTNL/ BTN proteins may exert their impacts as heterodimers of Btnl $1+6$, $\mathrm{BTNL} 3+8$, and BTN3A1 $+2 \mathrm{~A} 1$, respectively $16,19,20,25$. Nonetheless, the functional significance of heteromers has not been universally accepted ${ }^{26}$, with one concern being that the most compelling evidence is based on cellular over-expression systems ${ }^{27,28}$.

This study has addressed this important issue by use of genetics. By showing that $\mathrm{V} \gamma 5 \mathrm{~V} \delta 1^{+}$DETC development depends on Skint2 as well as on Skint1, and that $\mathrm{V} \gamma^{+}$intestinal IEL development depends on Btnl6 as well as on Btnl1, we now provide formal genetic evidence that single Btnls are not sufficient for IEL selection. Most unexpectedly, however, different Btnl pairings had differential effects on IEL with different TCRs, revealing a potential for combinatorial diversity that could finely tune IEL repertoire composition. The major impacts of Skint1 and Btnl1 on IEL maturation occur during narrow time-windows in early life. Beyond this, the sustained expression of Btnl genes is herewith shown to be required to maintain signature intestinal IEL phenotypes. In sum, epithelial Btnl proteins mediate a sustained and complex regulation of local $\gamma \delta$ T-cell compartments.

\section{Results}

DETC development requires Skint2. The normal, intrathymic development of $\mathrm{V} \gamma 5 \mathrm{~V} \delta 1^{+}$DETC progenitors depends on Skint1, as judged by severe DETC depletion in Skint1 hypomorphic (FVB.Taconic), Skint1-deficient $\left(\operatorname{Skint}_{1}{ }^{\Delta / \Delta}\right)[\Delta$ denotes internal deletion] or Skint locus deficient mice ${ }^{17,29,30}$. To ask whether DETC development depends on at least one other Skint gene, we used CRISPR to target Skint2, which seems evolutionarily conserved across rodents possessing DETC ${ }^{31}$. To disrupt Skint2, we introduced LoxP (fl) sites flanking the first and fifth proteincoding exons (exons 2 and 6). However, as is common in CRISPR strategies, a collateral outcome was an internal deletion spanning those exons (Supplementary Fig 1a). Those Skint $2^{\Delta / \Delta}$ mice showed no Skint2 mRNA expression in ear skin (Fig. 1a) or elsewhere, whereas wild-type (wt) Skint1 mRNA levels were sustained.

$\mathrm{V} \gamma 5 \mathrm{~V} \delta 1^{+}$DETC progenitors in the fetal thymus of $\sim \mathrm{E} 15.5 \mathrm{wt}$ mice show Skint1-dependent selective maturation, as indicated by CD45RB and CD122 upregulation and CD24 and CD62L downregulation $^{30}$. Conspicuously, $\mathrm{V} \gamma 5 \mathrm{~V} \delta 1^{+}$DETC progenitors in $S k i n t 2^{\Delta / \Delta}$ mice phenocopied those in Skint1 hypomorphs ${ }^{30}$ and Skint $^{\Delta / \Delta}$ animals ${ }^{29}$, failing to mature relative to coexamined wild-type (wt) controls, but showing compensatory increases in immature CD45RB ${ }^{l o}, \mathrm{CD} 122^{\mathrm{lo}}, \mathrm{CD} 24^{\mathrm{hi}}$ and $\mathrm{CD} 62 \mathrm{~L}^{+}$ cells (Fig. 1b, c). Unsurprisingly, this maturation defect resulted in almost complete loss of mature DETC expressing the 17D1 epitope displayed by the $\mathrm{V} \gamma 5 \mathrm{~V} \delta 1$ DETC TCR (Fig. 1d-f; Fig Supplementary Fig. 1b). The so-called DETC-replacement cells were TCR $\gamma \delta^{+}$, demonstrating that Skint2 deficiency did not cause pan- $\gamma \delta$ deficiency (Fig. 1d, e). Moreover, although they completely lacked 17D1 ${ }^{+}$DETC, some Skint $2^{\Delta / \Delta}$ mice harboured $\mathrm{V} \gamma 5^{+}$DETC-replacements (Fig. 1d-f) although their TCR expression was somewhat lower than wt $\mathrm{V} \gamma 5^{+}$DETC, symptomatic of defective selection ${ }^{18,30,32}$ (Fig. 1d, e). By contrast to the dramatic change in the DETC compartment, Skint $2^{\Delta / \Delta}$ mice showed a largely unchanged representation of MHC-class $\mathrm{II}^{+}$-Langerhans cells with which DETC share the epidermis (Fig. 1f; Supplementary Fig. 1b). The significance of these various phenotypic patterns notwithstanding, DETC and LC counts showed some inter-individual variation (Fig. 1f), indicative of the cells' multifactorial regulation, although there was no evident contribution of sexual dimorphism (Supplementary Fig. 1c).

The cells with a TCR most closely related to $\mathrm{V} \gamma 5 \mathrm{~V} \delta 1^{+}$DETC are uterine and lung $\gamma \delta$ T cells expressing $\mathrm{V} \gamma 6$ paired with a $\mathrm{V} \delta 1$ chain identical to that in DETC. In the absence of a generally available $\mathrm{V} \gamma 6$-specific antibody, such cells were identified as TCR $\gamma \delta^{+} \mathrm{V} \gamma 1^{-} \mathrm{V} \gamma 4^{-} \mathrm{V} \gamma 5^{-}$, and in Skint $2^{\Delta / \Delta}$ mice such cells were largely unaffected (Supplementary Fig. 1d,e), again phenocopying Skint1 hypomorphs ${ }^{30}$. Collectively, these genetic data show that Skint2 as well as Skint1 is critically required for the specific maturation of $\mathrm{V} \gamma 5 \mathrm{~V} \delta 1^{+}$DETC progenitors, supporting the hypothesis that discrete $\gamma \delta$ T-cell compartments are naturally regulated by Btnl heteromers.

Indeed, the capacity of Skint1 and Skint2 to form physical complexes in vitro and in vivo was validated when antiSkint 1 immunoprecipitates from 293 T cells transfected with Nterminal Flag-tagged Skint1 and HA-tagged Skint2 were shown to contain both Skint1 and Skint2, as detected by western blot (Supplementary Fig. 1f). Moreover, anti-Skint1 and anti-Skint2 antibodies could detect Skint1 and Skint2, respectively, in western blots of anti-Flag immunoprecipitates from thymi of transgenic mice expressing an N-terminal Flag-tagged Skint1 construct (NF-Skint1 $\left.{ }^{\mathrm{Tg}}\right)^{32}$, but not from non-transgenic FVB mice (Fig. 1g; long arrows). Note that the detection of anti-Flag antibody chains in the FVB lysates (Fig. 1g; asterisks) validated protein loading. Moreover, the specificity of Skint1 and Skint2 detection in the immunoprecipitates was verified by the failure to detect actin in anti-Flag immunoprecipitates, despite its detection in total input protein (Fig. 1g, lowest panel). The 
a

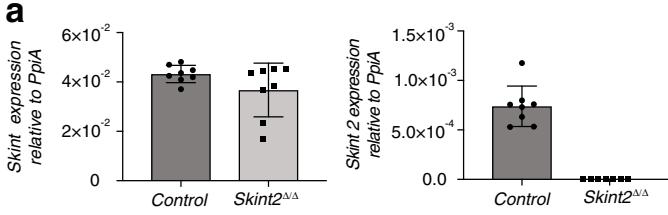

C
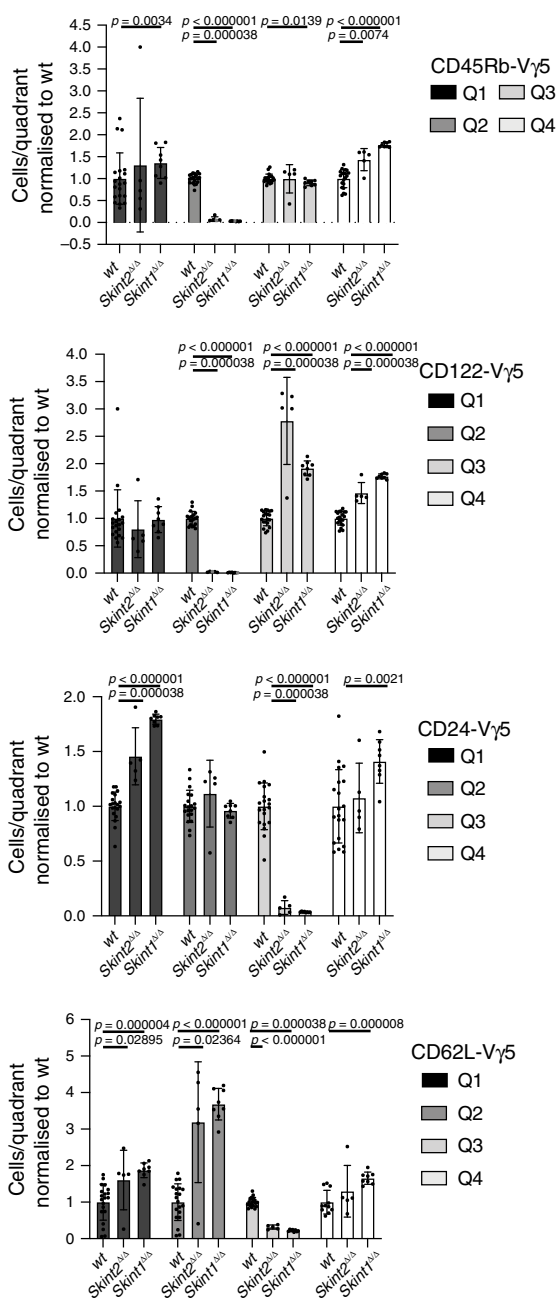

e

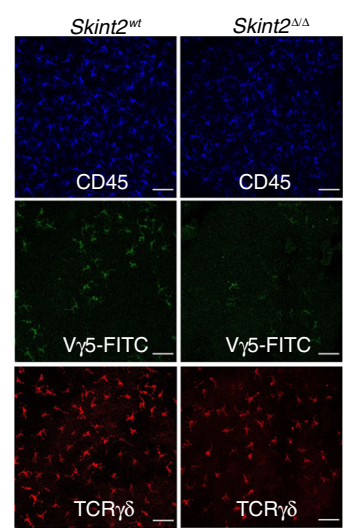

$\mathbf{f}$
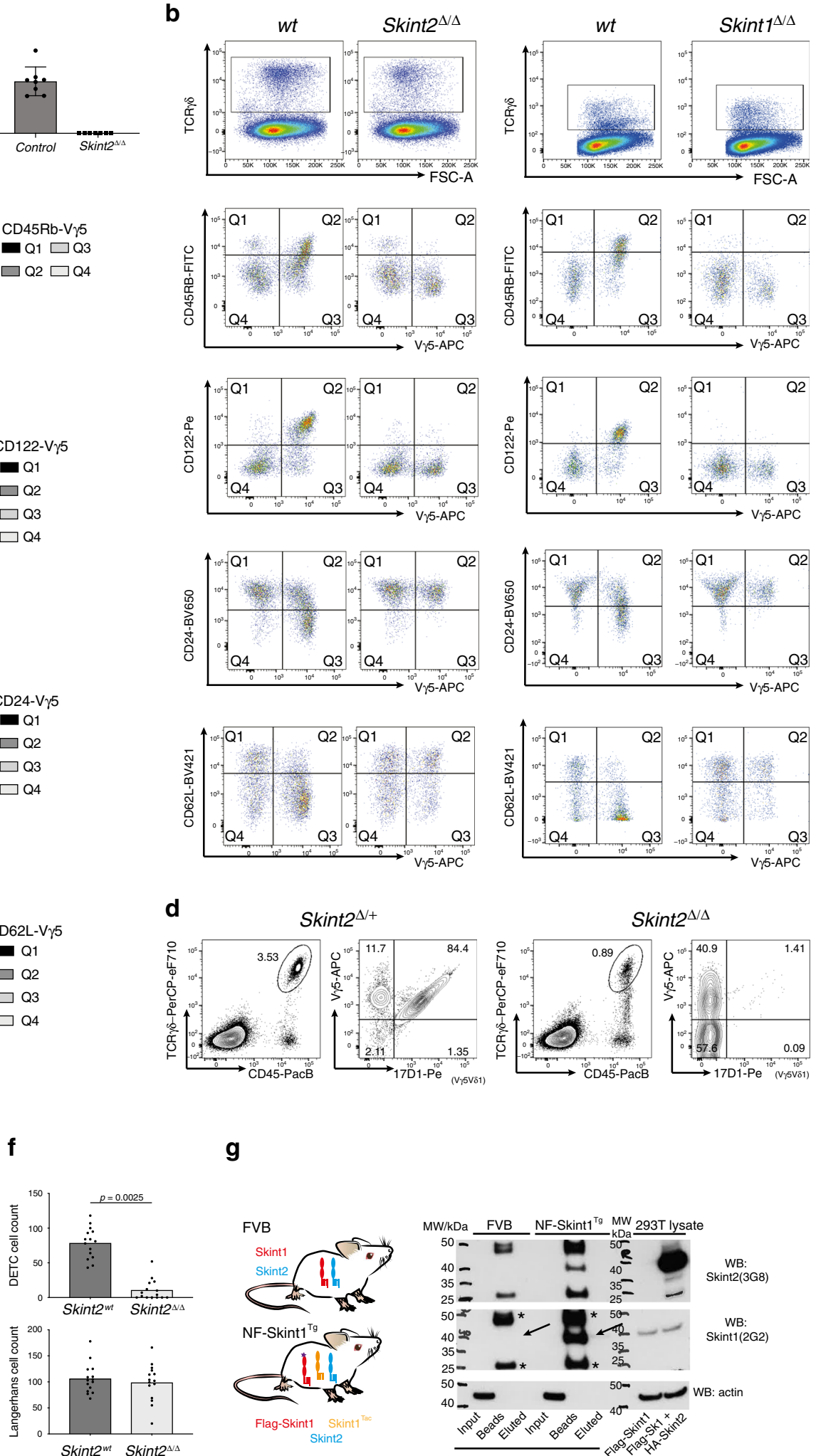

d

Skint2 ${ }^{\Delta+}$
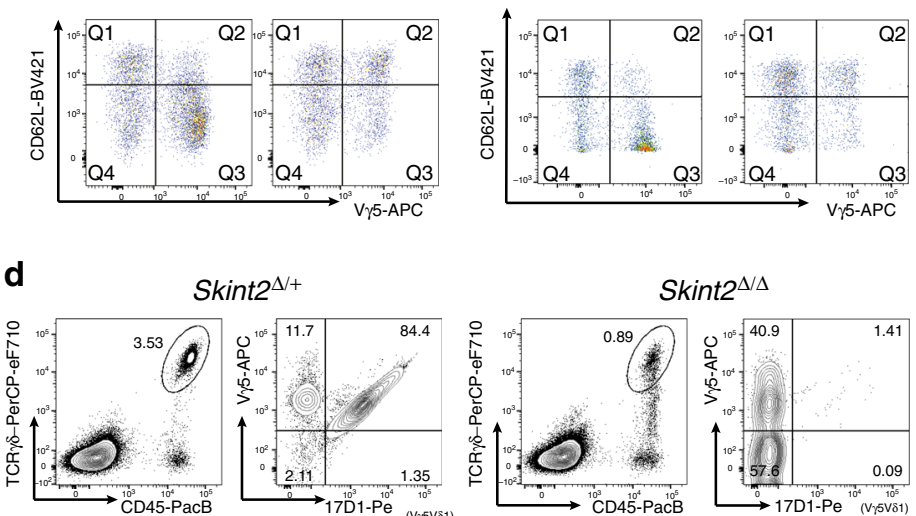

g
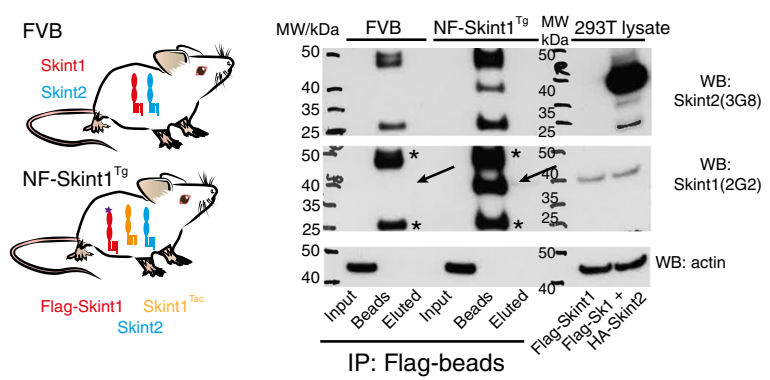

and Skint2 to associate in cell lines and in primary mouse tissue.

Btnl genes exert hierarchical regulation of $\mathrm{V} \gamma 7^{+}$IEL. Small intestinal villus enterocytes express Btnl1, Btnl4 and Btnl6 failure to detect Skint1 or Skint2 in total input protein is consistent with their very low levels of protein expression ${ }^{32}$. The inefficiency of Skint1/Skint2 elution from the beads seemingly reflects a greater affinity of the anti-Flag antibody for Flag-tagged Skint proteins versus Flag peptide. This notwithstanding, the data show an evident capacity of Skint 1 
Fig. 1 DETC development requires Skint2 and Skint1 which form heteromers. a qPCR analysis for Skint1 and Skint2 message in adult mouse ear epidermis normalised to Ppia. Control, $n=8$, Skint $2^{\Delta / \Delta}, n=8$. Data are mean \pm SD of a representative experiment of three independent experiments. $\mathbf{b}$ Analysis of E16.5 thymocytes in WT, Skint $2^{\Delta / \Delta}$ and $S k i n t 7^{\Delta / \Delta}$ animals, gated on live $\gamma \delta$ T cells (top panel). Thymic $\gamma \delta$ cells (gate top row) were assessed for CD45RB, CD122, CD24 and CD62L and expression of the $V \gamma 5$ TCR. Left panel: WT vs. Skint $2^{\Delta / \Delta}$, right panel: WT vs. Skint $7^{\Delta / \Delta}$. c Quantification of cell populations in quadrants Q1 to Q4 as indicated in (b), normalised to mean of wt $=1$ for each quadrant, mean \pm SD. WT $n=20 ; S k i n t 2^{\Delta / \Delta} n=5 ; S k i n t 7^{\Delta / \Delta} n=8$ (twotailed Man-Whitney analysis). d FACS analysis of ear epidermis in control (Skint2 $2^{\Delta /+}$ ) and Skint $2^{\Delta / \Delta}$ mice. CD45 ${ }^{+}$, TCR $\gamma \delta^{+}$cells (gate left panel) were stained for presence of $\mathrm{V} \gamma 5 \mathrm{~V} \delta 1^{+} \mathrm{DETC}$ (stained by $\mathrm{V} \gamma 5$ and 17D1 antibody, right panel). e Microscopy images of adult mouse ear epidermal sheets in control and Skint $2^{\Delta / \Delta}$ mice. Comparison of DETC stained for CD45 (top: blue) and V $\gamma 5^{+}$(middle: green) $\gamma \delta-\mathrm{TCR}\left(\mathrm{GL} 3^{+}\right.$) cells (bottom: red). Scale bar $50 \mu \mathrm{m}$. f Quantification of DETC and Langerhans cell numbers from microscopy images, $n=15$ for each genotype (data are from three independent experiments, two-tailed Man-Whitney analysis). $\mathbf{g}$ Immunoprecipitation of Flag-tagged Skint1 from FVB or NF-Skint1Tg animals. Left: scheme of FVB mice expressing Skint1 and Skint2. Scheme: Top: wt FVB mice express endogenous, untagged Skint1 and Skint2; bottom: NF-Skint1 ${ }^{\top g}$ animals express a Flagtagged Skint1 and untagged Skint2 on the Skint $1^{\text {Tac }}$ background. Right: Immunoprecipitation with anti-Flag antibody from lysates of pooled thymi of FVB and NF-Skint1 ${ }^{\top g}$ animals ( $n_{\mathrm{FVB}}=12, n_{\mathrm{NFSkint1T}}=22,1$ experiment). Expression control in 293 lysates transduced with either Flag-Skint1 alone or Flag-Skint1 \& HA-Skint2 constructs. Long arrows: Skint1 band, asterisks: non-specific bands reflecting anti-FLAG Ig chain detection. Full scans are provided in Supplementary Fig. 7.

genes $^{16,33,34}$. Whereas Btnl1-deficient mice were substantially depleted of signature $\mathrm{V} \gamma 7^{+}$intestinal IEL, Btnl4 deficiency had no obvious effect ${ }^{16}$. Therefore, to test whether the heteromeric model also applied to the gut, we generated mice lacking Btnl6, which encodes a protein that can collaborate with Btnll to stimulate mature $\mathrm{V} \gamma^{+}$intestinal $\mathrm{IEL}^{16}$. To this end, we introduced loxP sites on either side of the 9-exon gene (Fig. 2a, right panel; $B t n l \sigma^{f l f l}$ mice). In parallel, to complement the Btnl1 ${ }^{K O}$ strain previously obtained from the International Knockout Mouse Consortium $\left(B t n l 1^{K O M P}\right)^{16}$, we generated a floxed allele of Btnl1 with LoxP sites flanking the first four coding exons (exons 2-5) (Fig. 2a, left panel). A constitutive, universal knockout of Btnl6 (Btnl6 $6^{\Delta / \Delta}$ mice) was generated by crossing floxed Btnl6 with PgkCre mice ${ }^{35}$, while intestinal epithelial cell (IEC)-specific knockouts of Btnl6 (Btnl6 ${ }^{\Delta g u t}$ mice) and Btnl1 (Btnl1 $1^{\Delta g u t}$ mice) were generated by crossing the floxed mice to Villin-Cre mice ${ }^{36}$ (Fig. 2a). The veracity of the different mutant mouse strains was evident from quantitative RT-PCR of Btnl1, Btnl4 and Btnl6 expression, and histologic RNAScope analysis of Btnl1 and Btnl6 (Supplementary Fig. 2a,b).

The intestinal IEL compartment of adult Btnl1 $1^{\Delta g u t}$ mice strikingly phenocopied the complete Btnl1 knockout, displaying substantial and significant reductions of $\mathrm{V} \gamma^{+}$cells, and of $\mathrm{V} \gamma 7 \mathrm{~V} \delta 4^{+}$cells that we previously showed to be particularly affected by Btnl1 deficiency ${ }^{16}$ : hence, the profound $\gamma \delta$-regulatory impact of Btnl1 seems attributable exclusively to IEC (Fig. 2b, c). Most unexpectedly, however, Btnl6 $6^{\Delta / \Delta}$ and $B t n l 6^{\Delta g u t}$ mice showed an overtly intermediate phenotype, with $\mathrm{V} \gamma^{+}$IEL and $\mathrm{V} \gamma 7 \mathrm{~V} \delta 4^{+}$ IEL significantly reduced relative to either C57BL/6 or Btnl $6 / / f l$ mice, as measured by a $33-50 \%$ decrease in their percentage representation among gut $\gamma \delta$ cells or by a 2 -fold drop in absolute numbers of $\mathrm{V} \gamma^{+}$IEL, by contrast to the cells' almost complete loss from Btnl1 ${ }^{\Delta g u t}$ mice (Fig. $2 \mathrm{~b}-\mathrm{d}$ ).

The few residual IEL in Btnl1 ${ }^{\Delta g u t}$ mice showed major dysregulation of the signature $\mathrm{V} \gamma 7^{+}$IEL phenotype, with many cells displaying low expression of CD122 (the IL-15R chain) and high Thy1 (CD90) expression (Fig. 2d, e; Supplementary Fig. 2c). Strikingly, such dysregulation was not true for Btnl6 ${ }^{\Delta u t}$ or $B t n l 6^{\Delta / \Delta}$ mice, in which residual IEL showed comparable phenotypes to controls (Fig. 2d, e; Supplementary Fig. 2c). Furthermore, whereas the $\mathrm{V} \gamma 7 \mathrm{~V} \delta 4$ TCR mean fluoresecence intensity (MFI) was somewhat lower in Btnl1-deficient mice, consistent with defective selection ${ }^{16}$, it was unaltered in Btnl6 $6^{\Delta u t}$ and $B t n l 6^{\Delta / \Delta}$ mice (see Fig. 2c). Hence, Btnll and Btnl6 differentially affected $\mathrm{V} \gamma 7^{+}$IEL development, with Btnl6 required for the normal size of the IEL compartment, but not for the acquisition of the signature phenotype by residual $\mathrm{V} \gamma 7^{+}$IEL. Moreover, the unique phenotype of $B \operatorname{tnl} \sigma^{\Delta / \Delta}$ mice was specific in that myriad immune subsets in the spleen were unaltered relative to controls (Supplementary Fig. 2d; Supplementary Table 1), as was reported for $B t n l 1^{-/-}$mice ${ }^{16}$.

To test whether those $\mathrm{V} \gamma 7^{+}$IEL that developed seemingly normally in Btnl6-deficient mice were Btnl-dependent, we generated mice lacking all three intestinal epithelial Btnls, by targeting sites upstream of the initiator ATG codons in Btnl1 and Btnl6, respectively (note that those genes are transcribed in head-to-head orientation), thereby deleting over $130 \mathrm{~kb}$ in between, including the Btnl4 gene (Fig. 2f). The resultant $\left(B t n l 146^{I N D E L}\right)$ mice expressed no detectable Btnl1, Btnl4 or Btnl6 transcripts, and there was also reduced expression of the Btnl2 gene that immediately flanks the deletion. Conversely, Psmb9, which is more distal to the recombination point was unaffected (Supplementary Fig. 2e).

When Btnl146 ${ }^{I N D E L}$ mice were contemporaneously compared with $B t n l 1^{\Delta / \Delta}, B t n l \sigma^{\Delta g u t}$ and Btnl4-deleted mice $\left(B t n l 4^{K O M P}\right)$ that we previously characterised ${ }^{16}$, it was clear that Btnl146 ${ }^{I N D E L}$ mice (colour-coded green in Fig. 2g-i) largely phenocopied the near-ablation of $\mathrm{V} \gamma 7^{+}$IEL in Btnl1deficient mice (Fig. $2 \mathrm{~g}, \mathrm{~h}$ ). In both cases, residual $\mathrm{V} 7^{+}$cells showed reduced $\mathrm{V} \gamma 7$ and V 84 TCR MFI and failed to upregulate CD122 or downregulate Thy1, by comparison to IEL in control, Btnl4 ${ }^{K O M P}$ or Btnl6-deficient strains (Fig. $2 \mathrm{~g}, \mathrm{i}$ ). When we integrated data from large numbers of mice of the different strains described, it became clear that the strains' respective $\mathrm{V} 7^{+}$IEL compartments were consistent and stable over time for $>120$ days (Supplementary Fig. 2f).

In sum, further support for the heteromer hypothesis was provided by the developmental dependence of approximately one-third to one-half of intestinal $\mathrm{V} 7^{+}$IEL on Btnl6 as well as Btnl1. Nonetheless, an unanticipated nuance was introduced in that there was a hierarchy of Btnl regulation, with V $\gamma 7$ IEL numbers depending almost completely on Btnl1, partially on Btnl6, and not on Btnl4, while the signature phenotypes of $\mathrm{V} 7^{+}$ IEL present in the different strains were largely dependent on Btnl1, but independent of either Btnl6 or Btnl4.

Btnl6 deficiency alters $\mathrm{V} \delta$ gene usage. Although $\mathrm{V} \gamma 7$ usage denotes the signature intestinal $\gamma \delta$ IEL compartment, $V \delta$ usage is also limited to some degree, with V $\delta 4$ (encoded by the $T r d v 2-2$ gene) and V $\delta 7$ predominating, whereas $\leq 10-15 \%$ of $\mathrm{V} \gamma 7^{+}$cells express TCR V $\delta 6.3$ (encoded by identical $T r d v 6 D-1$ and $\operatorname{Tr} d v 6 N$ 1 genes) (Fig. 3a) ${ }^{19}$. Conversely, slightly more $\mathrm{V} \gamma 7^{(-)}$IEL in wt mice expressed V86.3, although the TCR MFI was lower vis-à-vis $\mathrm{V} \gamma 7^{+} \mathrm{V} \delta 6.3^{+}$cells, typical of unselected cells (Fig. 3a, top panels).

By contrast, V86.3+ cells with high TCR MFI accounted for $>30 \%$ of residual $\mathrm{V} 7^{+}$IEL in both Btnl6-mutant strains, a highly 
a

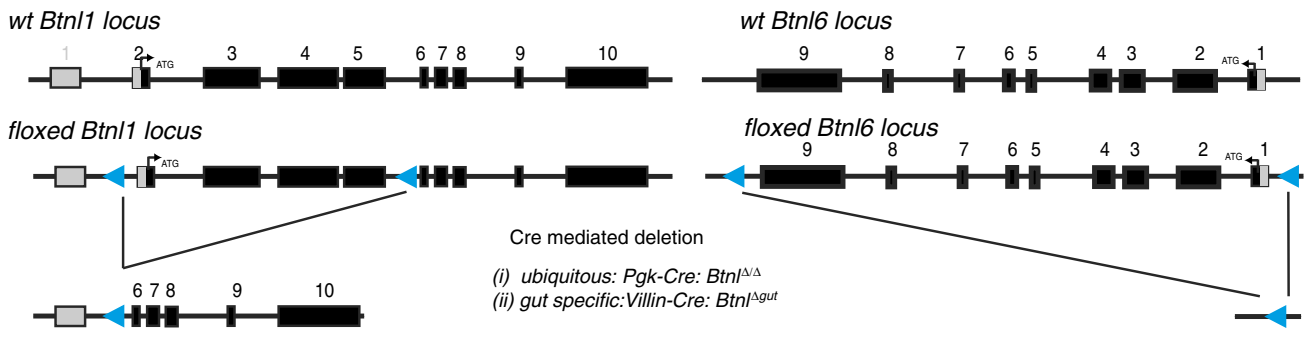

b

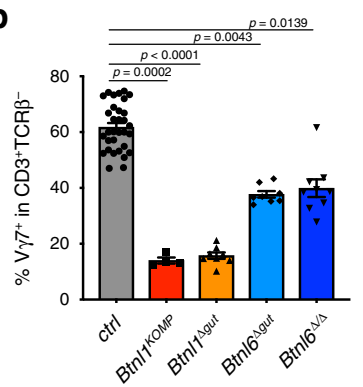

d
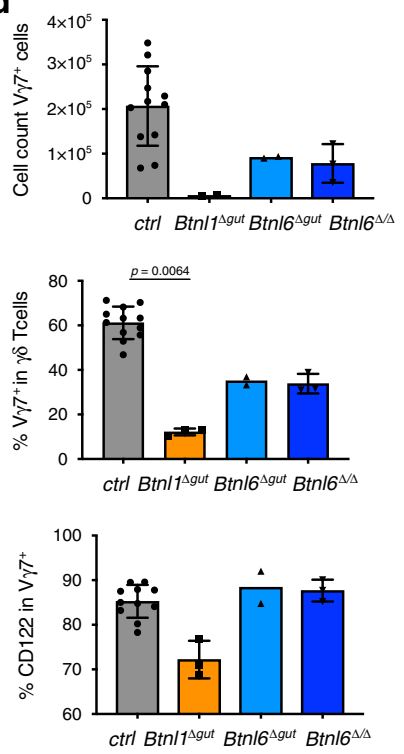

e

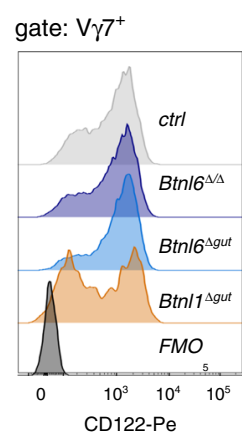

h
C

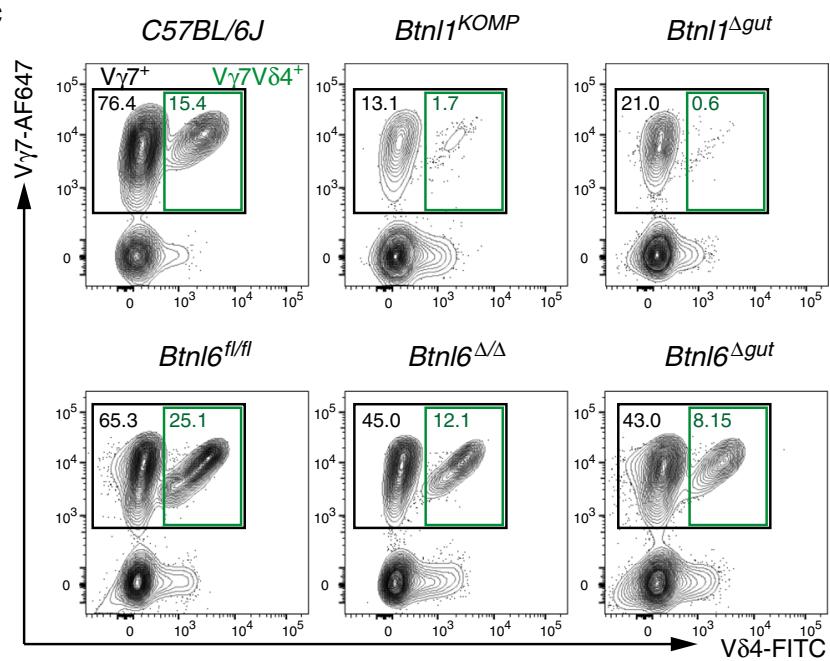

f

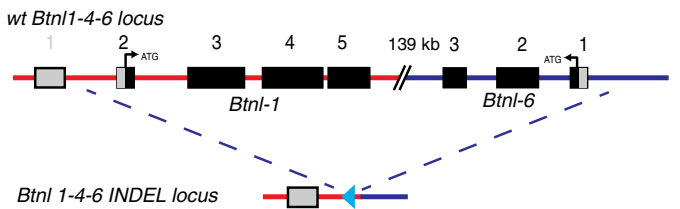

$g_{\mathrm{TCR} \beta}{ }^{\mathrm{CDD}} 3^{+}$
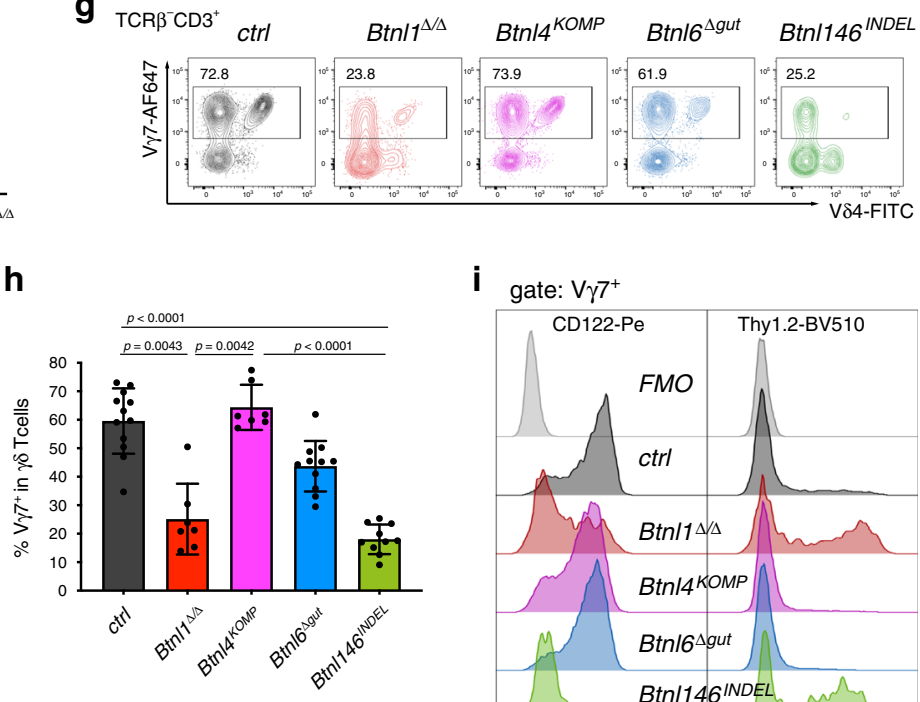

i

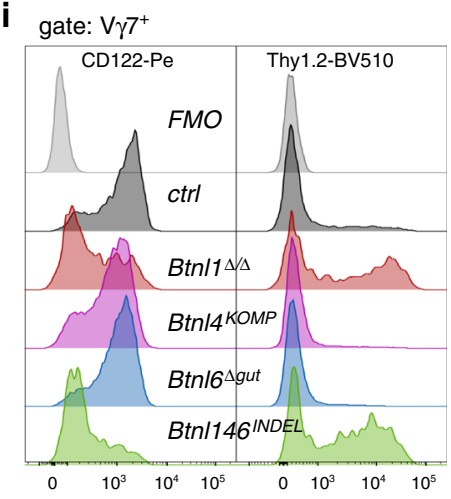

significant difference from controls (Fig. 3a-c, Supplementary Fig. 3). This unanticipated finding reflected the fact that $\mathrm{V} \gamma 7^{+} \mathrm{V} \delta 6.3^{+}$IEL numbers were essentially unaltered in Btnl6 $6^{\Delta u t}$ and $B \operatorname{tn} l 6^{\Delta / \Delta}$ mice versus wt mice, despite total $\mathrm{V} \gamma 7^{+}$IEL being reduced by $\sim 2$-fold (above) (Fig. $3 \mathrm{~b}$ ). Although some Btnl1 ${ }^{\Delta g u t}$ mice showed small increases in the percentage of $\mathrm{V} \delta 6.3^{+}$cells among $\mathrm{V} 7^{+}$IEL (Fig. 3a, bottom plots; Supplementary Fig. 3), the absolute numbers of $\mathrm{V} \gamma 7^{+}$IEL in this strain were so neglible as to make such comparisons somewhat unreliable (Fig. 3b). Indeed, the very few residual $\mathrm{V} \gamma 7^{+}$IEL in Btnl146 ${ }^{I N D E L}$ mice showed no significant increases in V $\delta 6.3$ representation, although there was some reduction in V $\delta 4$ usage (Fig. 3c, Supplementary Fig. 3a). In sum, $\mathrm{V} \gamma 7^{+} \mathrm{V} \delta 6.3^{+}$IEL showed essentially no requirement for Btnl6, by contrast to their dependence on Btnl1. 
Fig. 2 The intestinal IEL compartment is shaped by expression of distinct Btnl molecules. a Targeting strategy to generate conditional and constitutive Btnl1 and Btnl6 knockout mice. Depending on the Cre-transgenic strain used (i/ii) resulting animals are either ubiquitously deleted for the respective Btnl gene $(\Delta / \Delta)$, or harbour a tissue-specific deletion ( $\Delta$ gut). Black: translated exons, grey: untranslated regions, blue triangles: loxP sites. $\mathbf{b}$ Quantification of $\mathrm{V} \gamma 7^{+}$IEL (black gate in c) in Btnl1 and Btnl6-deficient strains. Data are mean \pm SEM of $\geq 2$ independent experiments. $n_{\text {ctrl }}: 32, n_{\text {Btnl1-KOMP: }} 4, n_{\text {Btnl11 } \Delta \text { gut }}: 8$,

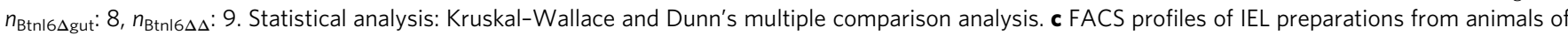
indicated genotypes, gated on TCR $\gamma \delta^{+}$cells. The label Btnl/ ${ }^{\mathrm{Komp}}$ indicates germline Btn/7 ${ }^{\mathrm{KO}}$ animals generated by the IMPC that have been described ${ }^{16}$. Black gate: all $\vee \gamma 7^{+}$cells, green gate: $\vee \gamma 7^{+} \mathrm{V} \delta 4^{+}$cells. $\mathbf{d}$ Quantification of IEL: $\vee \gamma 7^{+} \mathrm{IEL}$ numbers (top), \% $\gamma \gamma 7^{+} \mathrm{IEL}$ (middle) and \%CD122 ${ }^{+}$cells (bottom)

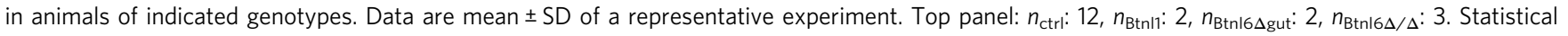

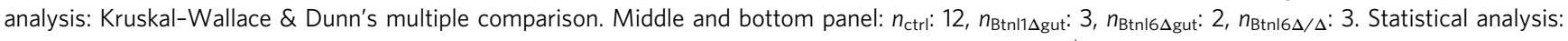
Kruskal-Wallace \& Dunn's multiple comparison. e Histogram for surface expression of CD122 in $V \gamma 7^{+}$IEL from animals of the indicated genotypes. f Scheme depicting the strategy to generate Btn/146 Indel mice. Short guide RNAs flanking the $5^{\prime}$ region of Btnl1 and $5^{\prime}$ region of Btnl6 were injected with HDR templates. Due to the nature of CRISPR/Cas9 the intervening region was excised and a Btn/146INDEL mouse lacking the Btn/7-4-6 locus was created. Blue triangle: loxP site that was inserted due to the nature of the HDR template (see Methods). $\mathbf{g}$ FACS analysis of TCR $\beta^{-} \mathrm{CD} 3^{+} \mathrm{IEL}$ in Btnl1/4/6-KO and Btn/146 INDEL mice. Colours correspond to coloured bar graphs in (h) and (i). $\mathbf{h}$ Quantification of $\vee \gamma 7^{+} \mathrm{IEL}$ depicted in $(\mathbf{g})$. Data are mean $\pm \mathrm{SEM}$ of three

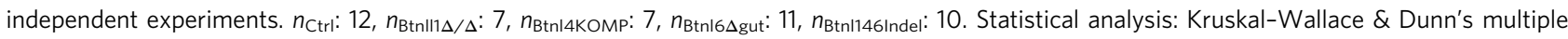
comparison. i Surface expression of CD122 (left) and Thy1.2 (right) in $V \gamma 7^{+}$IEL from animals of indicated genotypes.

We therefore investigated whether $\mathrm{V} \gamma 7^{+} \mathrm{V} \delta 6.3^{+}$might be regulated by a Btnll + Btnl 4 heteromer.

$\mathrm{V} \gamma 7^{+}$IEL respond to different Btnl combinations. Biochemical and molecular evidence has shown that Btnll + Btnl6 function is mediated by Btnl6 engaging $V \gamma 7$, while Btnll acts as a critical chaperone ${ }^{19,37}$. Btnl4 has near-identity to Btnl6 in the region (CFG) that engages $\mathrm{V} \gamma 7$ and both are diverged from Btnl1 (Fig. 4a, colour-coded orange, blue and red). To interrogate whether Btnl4 might substitute for Btnl6, we subjected primary IEL to co-culture with the MODE-K enterocyte cell line expressing either Btnl1 + Btnl6 (L1L6) or Btnl1 + Btnl4 (L1L4). The former pairing induced TCR downregulation and CD25 upregulation in ${\mathrm{V} \gamma 7^{+}}^{+}$IEL from wt mice ${ }^{16,19}$ (Supplementary Fig. 3b; top row, right panel), relative to which a significant albeit reduced effect was also induced by Btnll + Btnl4 (Supplementary Fig. 3b; top row, centre panel).

This response was further investigated by co-expressing Btnl1 in 293T cells with Btnl4 alleles mutated in each of three regions whose counterparts in Btnl6 are implicated in $\mathrm{V} \gamma 7$ engagement (Fig. 4a) ${ }^{19}$. Specifically, the Btnl4 sequences were replaced by counterparts from Btnll, in one case via the substitution of a single amino acid. Those mutations essentially ablated the TCR downregulation and CD69 upregulation ordinarily induced by Btnl1 + Btnl4 in a human T-cell line, J76, expressing a monoclonal V $\gamma 7 \mathrm{~V} \delta 4$ TCR (Fig. 4b). Thus, Btnl4 can phenocopy Btnl6 in co-operating with Btnl1 to regulate $\mathrm{V} 7^{+}$IEL, albeit that no IEL depend on Btnl4 for their maturation, as shown above.

To further examine responses to Btnl1 +4 versus Btnl1 +6 , we examined CD25 upregulation by primary $\mathrm{V} 7^{+}$IEL from different Btnl mutant strains (Fig. 4c). $V \gamma 7^{+}$IEL from wt mice were phenocopied by $\mathrm{V} 7^{+}$IEL from Btnl4 ${ }^{-1-}$ mice, and by those few residual $\mathrm{V} \gamma^{+}$cells in $B t n l 1^{-1-}$ mice in responding better to MODE-K cells expressing Btnl1 +6 compared with those expressing Btnll +4 . However, this was not true for $\mathrm{V}_{\gamma} 7^{+}$ IEL from Btnl6-deficient mice, which responded comparably or better to Btnl1 +4 (Fig. 4d; Supplementary Fig. 3b). Likewise, the very few residual $\mathrm{V} 7^{+}$IEL in Btnl146 $6^{I N D E L}$ mice showed comparable responses to Btnll +4 and Btnll +6 (Fig. 4d; Supplementary Fig. 3b).

When the responding $\mathrm{V} \gamma^{+}$IEL were further scrutinised, it became clear that in $\mathrm{wt}$ and $B t n l 4^{-1-}$ mice, the striking discrimination between Btnl1 +6 and Btnl1 +4 largely reflected the responses of $\mathrm{V} \gamma 7 \mathrm{~V} \delta 4^{+}$and $\mathrm{V} \gamma 7 \mathrm{~V} \delta 4^{-} \mathrm{V} \delta 6.3^{-}$IEL (Fig. 4e, left panel; Supplementary Fig. 3c, top two rows; Supplementary Fig. 3d). By contrast, largely comparable responses to Btnl $1+6$ and Btnl $1+4$, respectively, were made by $\mathrm{V} \gamma 7 \mathrm{~V} \delta 6.3^{+}$
IEL (Fig. 4e, right panel; Supplementary Fig. 3c, bottom two rows), which are over-represented in Btnl6 ${ }^{-I-}$ mice in which Btnl-dependent selection would of necessity be driven by Btnll + 4. In fact, $\mathrm{V} \gamma 7 \mathrm{~V} \delta 6.3^{+}$IEL, V $\gamma 7 \mathrm{~V} \delta 4^{+}$IEL and V $77 \mathrm{~V} \delta 4^{-} \mathrm{V} \delta 6.3^{-}$ IEL (which are mostly $\left.V \delta 7^{+}\right)^{19}$ from Btnl6 ${ }^{-1-}$ mice all showed relatively strong responses to Btnll +4 (Fig. 4e; Supplementary Fig. 3b,c), possibly consistent with their having been selectively expanded and matured by some combination of Btnl4 and Btnll.

To further investigate ${\mathrm{V} \gamma 7^{+}}^{+}$IEL regulation by Btnl1, Btnl4 and Btnl6, we expressed each separately and in combination in 293T cells. Surface display of Btnl6 was highly inefficient, but was rescued by co-expression of Btnll (Supplementary Fig. 4a). Conversely, Btnl4 alone could travel to the cell surface (Supplementary Fig. 4a): hence, Btnl6 and Btnl4 are not strictly comparable. ${\mathrm{V} \gamma 7^{+}}^{+} \mathrm{J} 76$ transductants (see above) were strongly stimulated by cells co-expressing Btnll +6 but not by cells expressing Btnl6 alone, nor by an admixture (Btnl1/Btnl6 sep) of cells expressing Btnl6 with cells expressing Btnll (Supplementary Fig. 4b, red). Conversely, 293T cells transduced with Btnl4 alone showed some capacity to stimulate $\mathrm{V} \gamma 7^{+} \mathrm{J} 76$ cells, although this was clearly increased by co-expressing Btnl1, but not by stimulating with an admixture of cells separately expressing Btnl4 and Btnll (Supplementary Fig. 4b, blue). These data evoke the activity of human BTNL3, a human $\mathrm{V} \gamma 4^{+}$-TCR ligand, which alone can provoke human $\mathrm{V} 4^{+}$-TCR downregulation, but whose effects are greatly amplified by BTNL8 co-expression ${ }^{19}$.

In sum, Btnl4 is evidently not required for $\mathrm{V} 7^{+}$IEL selection, but its capacity to stimulate $\mathrm{V} \gamma 7^{+}$IEL in vitro likely explains its capacity to select IEL, primarily $\mathrm{V} 7^{+} \mathrm{V} \delta 6.3^{+}$, in Btnl6-deficient mice. The starkly different phenotypes of Btnl6-deficient and Btnl1-deficient mice, argues that any Btnl4-intrinsic capacity to select $\mathrm{V} 7^{+}$IEL relies in vivo on co-expression with Btnll. Added to this, our data show that the signature preferential responses of $\mathrm{V} \gamma 7 \mathrm{~V} \delta 4^{+}$cells to Btnl1 +6 was seen only in cells from mice in which Btnl6 was expressed. This conditioning might be enforced during developmental selection, and/or be maintained in the steady-state by Btnl heteromers expressed in epithelial cells that juxtapose mature IEL. However, there has not hithtero been formal evidence of a maintenance function for Btnl genes, beyond their roles in selection. We therefore investigated this by use of conditional knockout mice.

Phenotypic maintenance by Btnl1 and Btnl6. We crossed floxed Btnll and Btnl6 strains to tamoxifen-regulated Villin-Cre mice, in order to generate mice in which Btnll and Btnl6 were inducibly deleted in IEC. Indeed, there was sustained loss of Btnll and Btnl6 expression, as assessed by RNAscope at 8 days and 22 days 
a

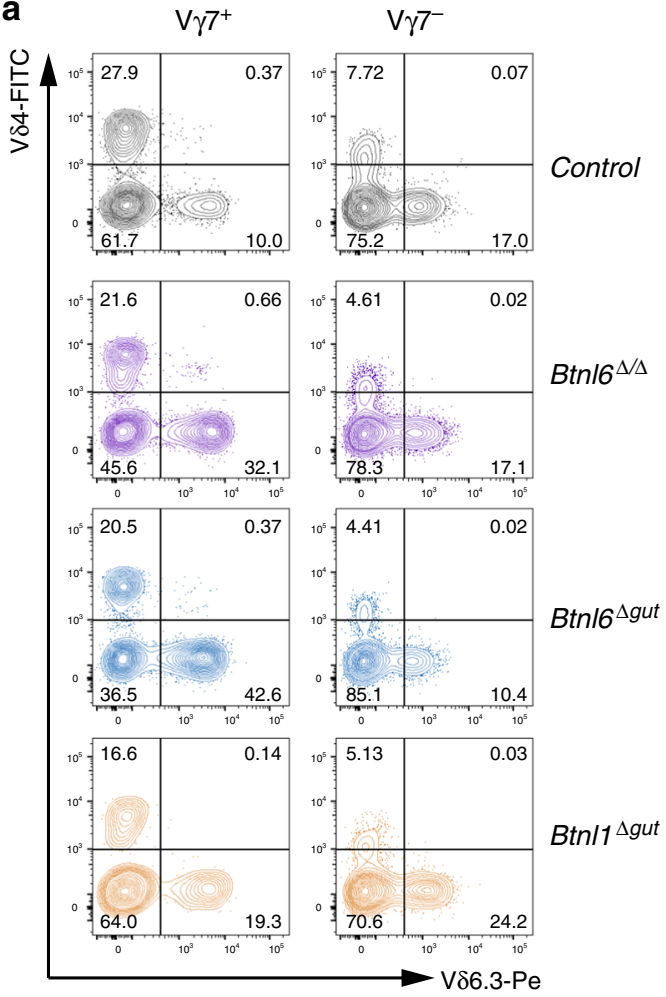

b
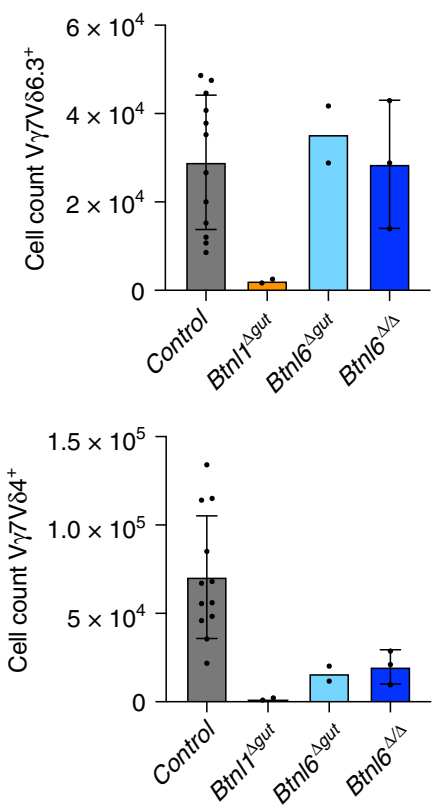

c

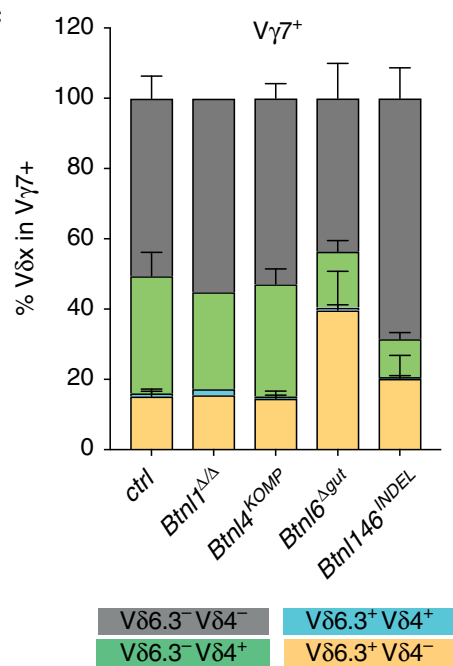

\begin{tabular}{|c|c|c|c|c|c|}
\hline \multicolumn{2}{|c|}{ compared groups } & V $\delta 6.3^{+} \mathrm{V} \delta 4^{-}$ & $\mathrm{V} \delta 6.3^{+} \mathrm{V} \delta 4^{+}$ & $\mathrm{V} \delta 4^{+} \mathrm{V} \delta 6.3^{-}$ & V $\delta 6.3^{-} \mathrm{V} \delta 4^{-}$ \\
\hline ctrl & $B \operatorname{tn} / 1^{\Delta / \Delta}$ & ns & ns & ns & ns \\
\hline ctrl & $B \operatorname{Bt} / 4^{\text {KOMP }}$ & ns & ns & ns & ns \\
\hline ctrl & $B t n / 6^{\Delta g u t}$ & $p<0.0001$ & ns & $p<0.0001$ & ns \\
\hline ctrl & $B t n / 146^{I N D E L}$ & ns & ns & $p<0.0001$ & $p<0.0001$ \\
\hline$B \operatorname{tn} / 1^{\Delta / \Delta}$ & $B t n / 4^{\text {KOMP }}$ & ns & ns & ns & ns \\
\hline$B \operatorname{tn} / 1^{\Delta / \Delta}$ & Btn/6 $6^{\Delta g u t}$ & $p<0.0001$ & ns & ns & ns \\
\hline$B \operatorname{tn} / 1^{\Delta / \Delta}$ & $B t n / 146^{I N D E L}$ & ns & ns & $p=0.0104$ & ns \\
\hline$B \operatorname{tn} / 4^{K O M F}$ & ${ }^{P} B$ Btnl6 $6^{\Delta g u t}$ & $p<0.0001$ & ns & $p=0.0025$ & ns \\
\hline Btn/4 КОМF & ${ }^{P} B t n / 146^{I N D E L}$ & ns & ns & $p<0.0001$ & $p=0.0058$ \\
\hline Btnl6 $6^{\Delta g u t}$ & $B \operatorname{tn} / 146^{I N D E L}$ & $p<0.0001$ & ns & ns & $p<0.0001$ \\
\hline
\end{tabular}

Fig. 3 Btnl6 deficiency alters V $\delta$ gene usage. a FACS plots of TCR $\delta$ chain usage in animals of indicated genotypes. The V $\delta 4$ and V $\delta 6.3$ chains are plotted against each other in $\mathrm{V} \gamma 7^{+}$IEL (left column) and $\mathrm{V} \gamma 7^{-}$cells (right column). b Quantification of $\mathrm{V} \gamma 7^{+} \mathrm{V} \delta 6.3^{+}$(top) and $\mathrm{V} \gamma 7^{+} \mathrm{V} \delta 4^{+}$cell numbers (bottom), in animals of indicated genotypes. $n_{\mathrm{ctrl}}: 12, n_{\mathrm{Btn} \mid 1}: 2, n_{\mathrm{Btn} \mid 6 \Delta \mathrm{gut}}: 2, n_{\mathrm{Btn} \mid 6 \Delta / \Delta}: 3$. Data are mean \pm SD of a representative experiment. c Quantification of $V \delta$ -

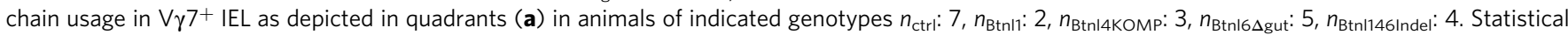
analysis: two-way ANOVA \& Tukey's multiple comparison post test. Data are mean \pm SD of a representative experiment.

following the start of 5 days' of tamoxifen administration (Fig. 5a), whereas there was no effect of tamoxifen treatment on mice lacking the relevant Cre allele (Fig. 5a; middle column). Durable loss of Btnl1 and Btnl6 expression suggested that gene deletion had occurred in enterocyte stem cells, as reported ${ }^{36}$. Over a 15-day period post tamoxifen-mediated Btnl1/Btnl6 deletion, no significant reduction was apparent in the representation of $\mathrm{V} \gamma 7^{+}$intestinal IEL, particularly by comparison to the reduced numbers seen in constitutively deleted $B \operatorname{tnl} 6^{\Delta / \Delta}$ mice and $B \operatorname{tnl} 1^{\Delta / \Delta}$ mice (Fig. 5b). Thus, signature IEL could be maintained at steady-state for at least two weeks in the absence of either Btnl1 or Btnl6.
Nonetheless, to investigate whether there might be more immediate effects of Btnl1/Btnl6 deletion, we applied tamoxifen daily for 3 days, and examined IEL 3 days later (Fig. 5c). Within this short time-frame, CD122 expression was markedly reduced on a large percentage of $\mathrm{V} 7^{+}$IEL in both Btnl1 ${ }^{f l f l} V c r e^{E R T 2+}$ and $B t n l f l l f l V c r e^{E R T 2+}$ mice (Fig. 5d). While this echoed the limited expression of CD122 by residual $\mathrm{V} \gamma 7^{+}$IEL in constitutive Btnl1 ${ }^{-1-}$ mice, it seemed a priori to conflict with sustained CD122 expression in constitutive Btnl6 ${ }^{-1-}$ mice (above). This conflict, however, was resolved by the finding that in mice acutely depleted of Btnl6, rapid CD122 downregulation was mostly limited to approximately half of $\mathrm{V} \gamma 7 \mathrm{~V} \delta 4^{+}$IEL (Fig. 5d). Indeed, $\mathrm{V} \gamma 7 \mathrm{~V} \delta 6.3^{+}$IEL (that are 
a

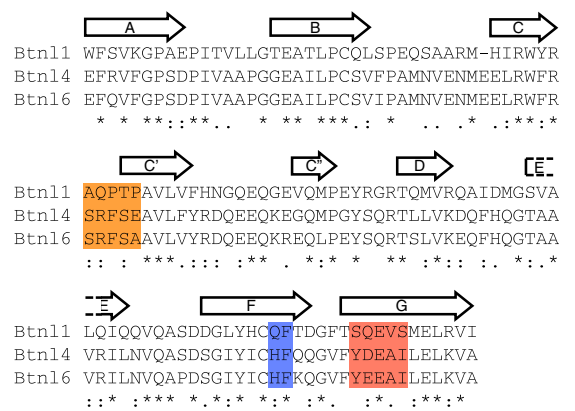

b
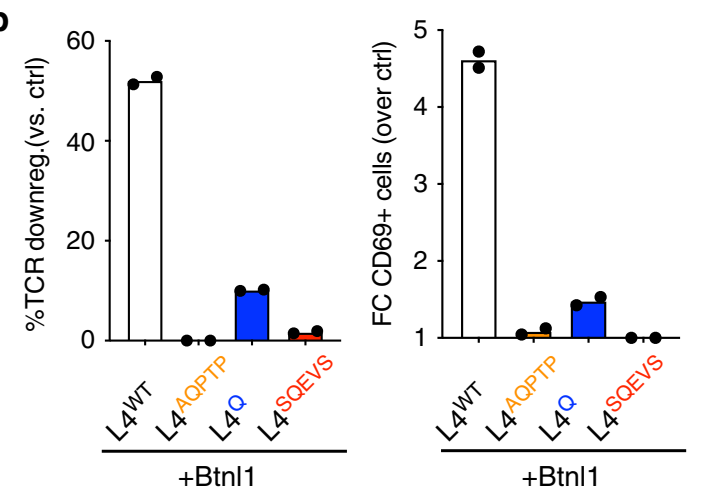

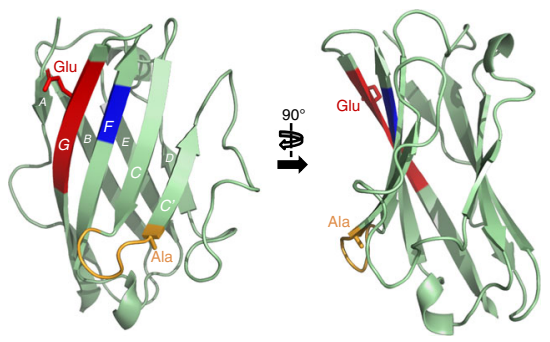

c
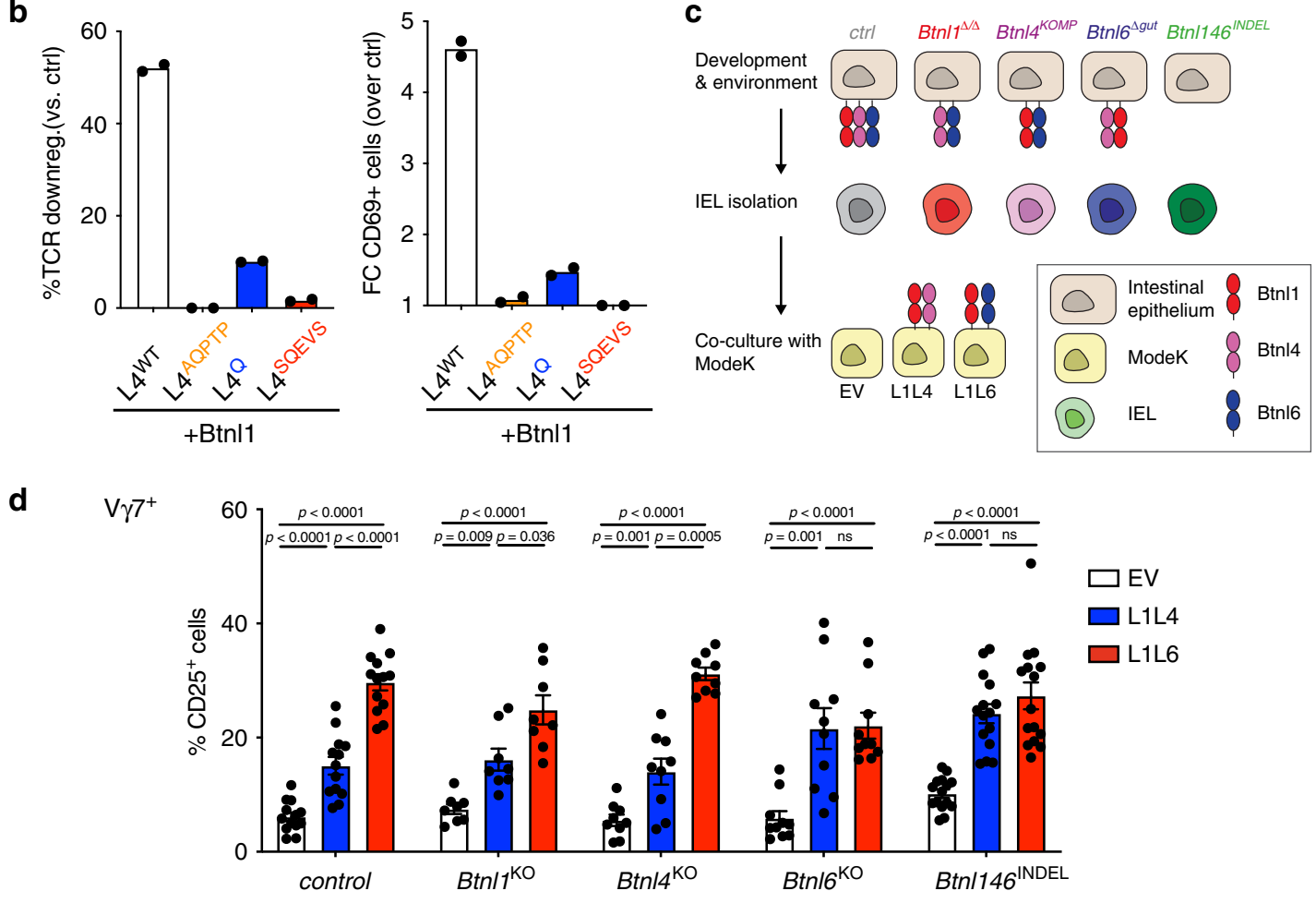

e

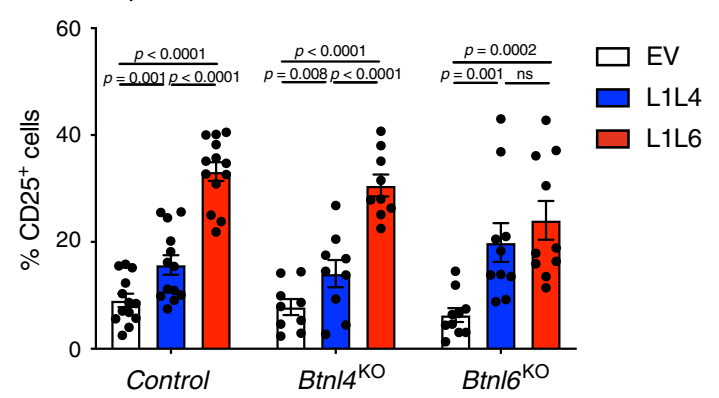

V $\gamma 7 \mathrm{~V} \delta 6.3^{+}$

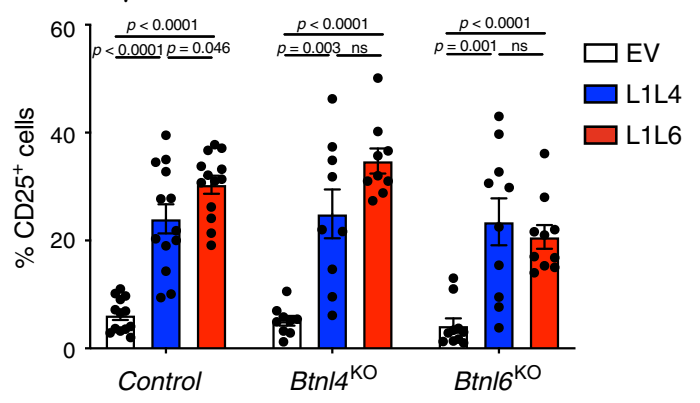

disproportionately enriched in constitutive $B t n l 6^{-1-}$ mice) were much less affected by acute depletion of Btnl6 versus Btnl1 (Fig. 5d), providing another example of the differential effects of Btnl proteins on different $\mathrm{V} \gamma^{+}$IEL subsets.

In this regard, we hypothesise that $\mathrm{V} \gamma 7 \mathrm{~V} \delta 6.3^{+}$IEL may have been selected on Btnl1 +4 even in wt mice, with their CD122 expression likewise maintained by Btnl1 +4 ; hence, they were essentially insensitive to acute Btnl6 depletion, phenocopying V $\gamma 7 \mathrm{~V} \delta 6.3^{+}$IEL and some V $\gamma 7 \mathrm{~V} 84^{+}$IEL in constitutive Btnl6 ${ }^{-1-}$ mice. Evidence in support of this hypothesis was provided by further analysis of $\mathrm{V} \delta$ usage by $\mathrm{V} \gamma 7^{+}$IEL, which was essentially unaffected at 3 days following Btnl6 depletion, but which was significantly skewed toward V86.3 ${ }^{+}$cells by day 56 (Fig. 5e, f). This would be consistent with natural IEL turnover favouring newly-maturing $\mathrm{V} \gamma 7 \mathrm{~V} \delta 6.3^{+}$IEL versus $\mathrm{V} \gamma 7 \mathrm{~V} \delta 4^{+}$IEL, since following Btnl6 deletion, the former could more efficiently engage Btnl1 + 4 .

The differential impacts of Btnl1, 4 and 6 on different IEL subsets might reflect their different spatio-temporal regulation. We therefore analysed single-cell RNA data available from studies in which distinct small intestinal populations were investigated. Consistent with our and others' studies $^{34,38}$, all three Btnls were restricted to enterocytes and enterocyte progenitors (Supplementary Fig. 5a), and spatially each peaked 
Fig. $4 \mathbf{V} \boldsymbol{\gamma} \mathbf{7}^{+}$IEL respond to different Btnl pairings. a Left: Alignment of the IgV-domain sequences of Btnl1, Btn|4 and Btnl6. Canonical Ig-fold $\beta$-strands $\left[A, B, C, C^{\prime}, C^{\prime \prime}, D, E, F, G\right]$ are indicated with arrows. CFG face motifs previously shown in Btnl6 to be critical for the response of $V \gamma 7^{+}$cells ${ }^{19}$ are highlighted in orange [AQPTP/SRFSE/SRFSA], blue [QF/HF/HF] and red [SQEVS/YDEAI/YEEAI]. Right: Cartoon representation of the IgV-domain of Btnl6, derived with 3D-JIGSAW from the crystal structure of BTN3A1 (PDB accession code 4F80), with the same annotation as in (a). Side chains are displayed for the two residues that differ in the CFG face motifs of Btnl6 versus Btnl4 (Ala versus Glu, Glu versus Asp). b TCR downregulation (left) and CD69 upregulation (right) by J76 cells expressing a V 7 V84 TCR and co-cultured with 293T transiently transfected with Btnl1 in combination with Btnl4 wild-type (L4WT) or mutated in the CFG region as indicated on the X-axis. Results are normalised to 293T transfected with empty vector (EV). Data are represented as mean \pm SD of duplicate co-cultures, representative of $n=2$ independent experiments. FC, fold change. c Experimental setup to analyse IEL from various $\mathrm{KO}$ strains in co-cultures with MODE-K cells overexpressing either Btnl1 and Btnl4 (L1L4) or Btnl1 and Btnl6 (L1L6). IELs are isolated from indicated mouse strains which can display distinct combinations of Btnl molecules on the epithelial surface during development. Following isolation, IEL were co-cultured o.n. with MODE-K cells displaying either Btnl1+4 or Btnl1+6 on their surface. MODE-K cells transduced with empty vector (EV) were used as control. d IEL response to MODE-K cells expressing different Btnl dimers (L1L4 or L1L6) was measured by analysing CD25+ cells gated on $V \gamma 7^{+}$

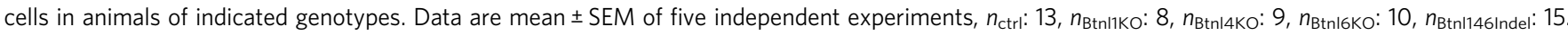
Statistical analysis: two-way ANOVA \& Tukey's multiple comparison post test. e IEL response to MODE-K cells expressing different Btnl dimers (L1L4 or L1L6) was measured as \%CD25+ cells and further gated on $\mathrm{V} \gamma 7^{+} \mathrm{V} \delta 4^{+}$(left) or $\mathrm{V} \gamma 7^{+} \mathrm{V} \delta 6.3^{+}$(right) cells in animals of indicated genotypes. Data are mean \pm SEM of five independent experiments, $n_{\text {ctrl }}: 13, n_{\text {Btnl4KO: }} 9, n_{\text {Btnl6ko: }} 10$. Statistical analysis: two-way ANOVA \& Tukey's multiple comparison post test.

in the middle of the basal-apical villus axis (regions $\mathrm{V} 2-\mathrm{V} 4$ ) (Supplementary Fig. 5b), aligning with the distribution of $\gamma \delta^{+}$ IEL $^{39}$. In sum, there was no obvious difference in spatiotemporal expression that might explain the proteins' differential effects, although there was an apparent hierarchy of RNA expression levels-Btnll $>>$ Btnl6 $>$ Btnl4-that evoked the hierarchy of the genes' effects on $\mathrm{V} 7^{+}$IEL.

Response maintenance by Btnl1 and Btnl6. To further investigate the requirement for sustained expression of Btnl genes, we examined IEL at 54 days after gut epithelium-specific deletion of the whole Btnl1,4,6 locus, making comparisons with wt mice and constitutive Btnl146 INDEL mice (Fig. 6a). (Note, acute loss of Btnl1,4,6 could not be examined because of variable penetrance of locus deletion until 1-month post tamoxifen treatment.) Locus loss for $\sim 8$ weeks again failed to diminish $\mathrm{V} \gamma 7^{+}$IEL numbers (Fig. 6b, middle panel, light green bars), supporting the conclusion that steady-state maintenance of Btnl-selected $\mathrm{V} \gamma 7^{+}$IEL numbers does not require sustained Btnl expression. Moreover, there was no significant increase in $\mathrm{V} \delta 6.3^{+}$cells consistent with there being no Btnl $1+4$ heteromers to promote their selective advantage (Fig. 6c). Interestingly, however, induced Btnl1,4,6 locus deletion also phenocopied constitutive Btnl146 ${ }^{I N D E L}$ mice in that the capacity of co-cultured $\mathrm{V} \gamma 7^{+}$IEL to respond preferentially to Btnll +6 versus Btnll +4 was lost over time (Fig. 6d). Diminished responses to Btnl1 +6 were seen for V $\gamma 7 \mathrm{~V} \delta 4^{+}$IEL and particularly for V $\gamma 7 \mathrm{~V} \delta 6.3^{+}$cells (Fig. 6e). This provides further support for the hypothesis that Btnl6 needs to be sustained to establish and to maintain the phenotype of cells that preferentially respond to Btnl $1+6$.

\section{Discussion}

$\gamma \delta$ T cells, particularly those residing within extralymphoid tissues, have been increasingly implicated in the regulation of tissue maintenance and protection against cancer ${ }^{39-44}$. Nonetheless, the cells' biologies remain poorly elucidated. Germane to this, a substantive advance was made by the discovery that different compartments of mouse and human $\gamma \delta$ T cells are critically and specifically regulated by butyrophilin and butyrophilin-like (Btnl) proteins. Moreover, recent cell biological, molecular and biochemical data fuelled the hypothesis that the active forms of Btnl proteins may be heterodimers, although there was heretofore no formal evidence supporting this in vivo. This study now provides genetic evidence for the importance of Skint/Btnl proteins functioning collaboratively, as would be the case for heteromers. In addition, our approach has revealed some surprising findings that emphasise the importance of genetics in understanding cell regulatory mechanisms.

Thus, signature murine skin $\gamma \delta$ IEL are shown to depend upon Skint $1+$ Skint 2 and the normal intestinal $\gamma \delta$ IEL compartment shown to depend upon Btnl1 + Btnl6. However, whereas Btnl1 ${ }^{\Delta / \Delta}$ mice lacked the great majority of $\mathrm{V} \gamma 7^{+}$IEL, $\sim 50 \%$ were retained in different strains of Btnl6-deficient mice. In seeking to understand this unanticipated hierarchy of Btnl proteins, we identified a potential of Btnl4 to substitute for Btnl6. However, whereas $\mathrm{V} 7^{+}$ IEL from wt mice ordinarily responded better in vitro to Btnl1 + Btnl6 versus Btnl1 + Btnl4, this was not so in Btnl6-deficient mice wherein the compartment of mature $\mathrm{V} \gamma 7 \mathrm{~V} \delta 6.3^{+}$and $\mathrm{V} \gamma 7 \mathrm{~V} \delta 4^{+}$ IEL, that was presumably selected by Btnl $1+$ Btnl 4 , responded comparably well to Btnl1 + Btnl4.

The CD $122^{\text {hi }}$ phenotype of most $\mathrm{V} \gamma 7^{+}$IEL was reduced when Btnl1 was acutely depleted, providing formal evidence that sustained expression of a $B t n l$ gene-product is required to maintain the signature status of the wt $\gamma \delta$ IEL compartment. By contrast, only a fraction of $\mathrm{V} \gamma 7^{+}$IEL showed CD122 downregulation when Btnl6 was acutely depleted. Moroever, the unaffected cells were enriched in $\mathrm{V} \delta 6.3^{+}$cells, phenocopying the repertoire composition in constitutive Btnl6-deficient mice. These data are consistent with the hypothesis that whereas Btnl4 is not required for the selection and/or maintenance of any $\mathrm{V} 7^{+}$IEL $^{16}$, some IEL in wt mice have naturally selected on Btnl1 + Btnl 4 while others selected on Btnl1 + Btnl6. Indeed, we propose that discrete Btnl heteromers ordinarily select those cells that respond most strongly to them and/or that they condition the responses of the cells they select. Thereafter, the Btnl heteromer on which the cells are selected is required to maintain the cells' signature phenotype. In sum, Btnl proteins operate in different combinations (i.e. show combinatorial diversity) in refining and regulating the composition of IEL compartments.

The biophysical basis for the preference of some cells for Btnl1 + Btnl6 versus Btnl1 + Btnl4 is unresolved. Btnl6 and Btnl1 physically associate, either directly or via an intermediate, and in this complex Btnl6 seemingly interacts directly with $\mathrm{V} \gamma 7^{37}$. Although there is currently less evidence available for the direct interaction of Btnl 4 with Btnl1, it is likely that the two associate given the Btnl1-dependence of essentially all $\mathrm{V} \gamma 7^{+}$IEL, and the capacity of Btnll co-expression to greatly increase the impact of Btnl4 on $\mathrm{V} 7^{+}$IEL. In this regard, the association of BTN2A1 and BTN3A1, which are jointly required to regulate human $\mathrm{V} \gamma 9 \mathrm{~V} \delta 2$ cells, only became evident after chemical cross-linking ${ }^{45}$. Nonetheless, some capacity of some mouse or human Btnl 
a
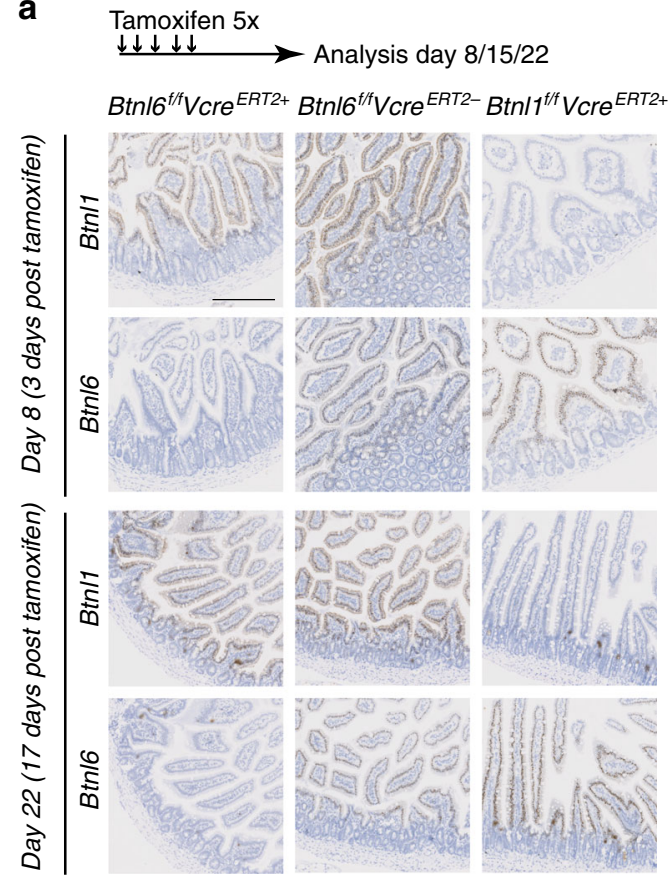

b

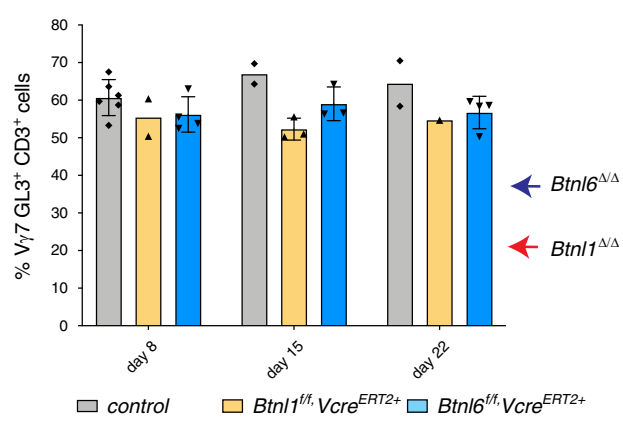

d

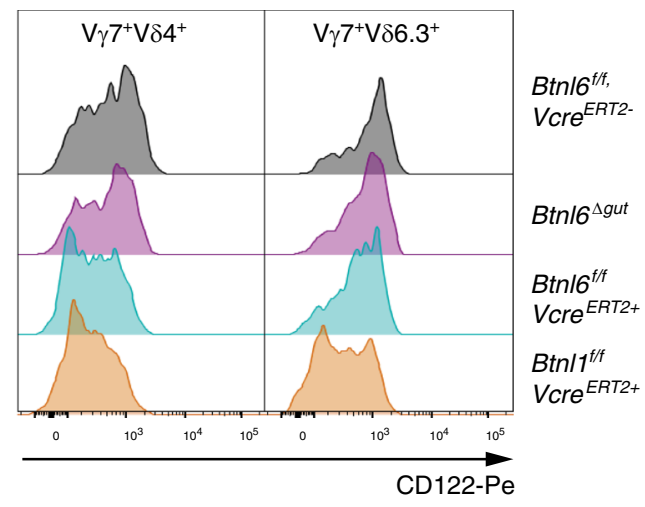

e

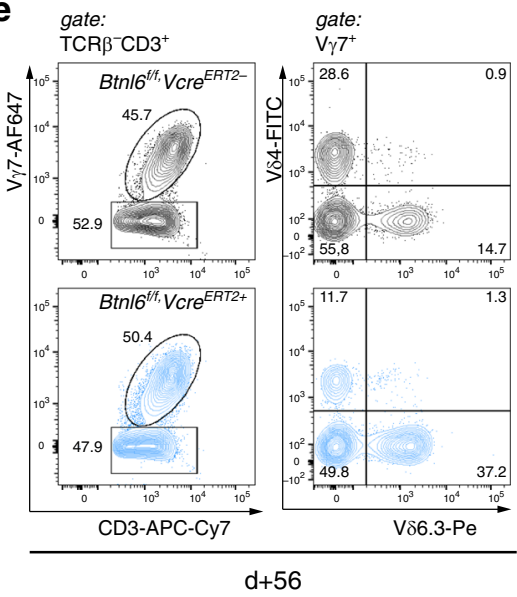

C

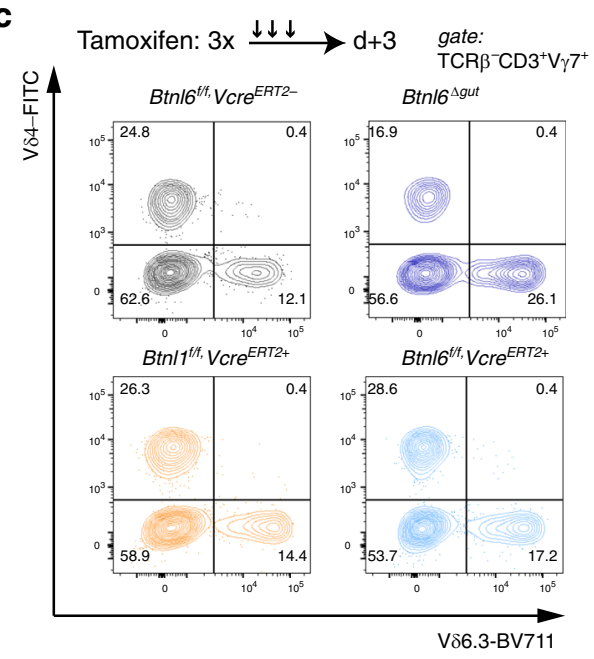

$\mathbf{f}$

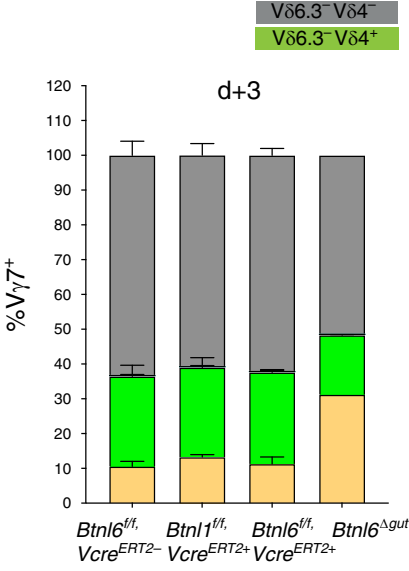

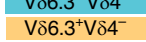

$120 \quad d+56$

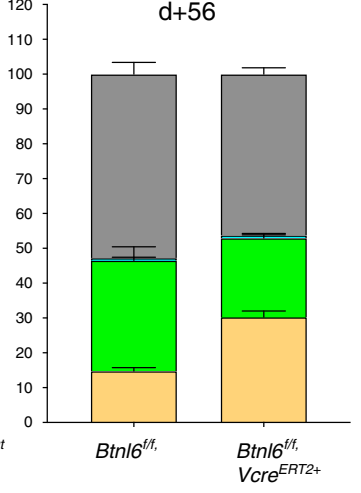

proteins (e.g. Btnl4; BTNL3), when over-expressed, to traffic to the cell surface and to regulate $\gamma \delta$ IEL, albeit suboptimally, leaves open the possibility that non-heteromeric complexes might be active, e.g., in disease settings in which BTNL proteins might be dysregulated.

Btnl1-dependence has to date been attributed solely to $\mathrm{V} \gamma 7$, and so an influence of $\mathrm{V} \delta$ chains on the Btnl response was a priori surprising. Possibly pairings with particular V $\delta$ chains might affect the response by altering the quarternary structures of TCRs. Alternatively, Btnl1 + 4-responsive IEL may comprise qualitatively distinct cells whose responsiveness might reflect their development along a distinct pathway: indeed, V $\delta 6.3$ expression has been associated with PLZF-expressing innate-like lymphocytes $^{46}$. This issue will be addressed by single-cell transcriptomics. 
Fig. 5 Depletion of individual Btnl genes does not impact $\mathbf{V} \boldsymbol{\gamma}$ IEL numbers but differentially affects CD122 expression. a Top: Experimental scheme to analyse the effect of Btnl1 and Btnl6 tamoxifen-mediated depletion at different timepoints. Bottom: RNAscope analysis for Btnl1 and Btnl6 in animals of indicated genotypes at 3 or 17 days post tamoxifen administration. Data are representative micrographs from one time course experiment with numbers of gut sections stained per genotype as: day 8: $n_{\text {ctrl: }}: 4, n_{\text {Btnl1f/f-VcreERT2+ }}: 2, n_{\text {Btnl6f/f-VcreERT2+: }}:$, day 22: $n_{\text {ctrl }}: 4, n_{\text {Btnllf/f-VcreERT2+- }}: 1, n_{\text {Btnl6f/f-VcreERT2+ }}: 2$, scale bar: $200 \mu \mathrm{m}$. b Quantification of $\vee \gamma 7^{+}$cells at indicated timepoints post tamoxifen (red and blue side arrows denote for comparison the average percentage of $V_{\gamma} 7^{+}$IEL in full knockout animals (see also Figs. 1-3). Data are mean \pm SD, day 8: $n_{\text {ctrl }}: 6, n_{\text {Btnllf/f-VcreeRT2+: }}: 2, n_{\text {Btnl6f/f-VcreERT2+: }}: 4$, day $15: n_{\text {ctrl }}:$ 2, $n_{\text {Btnl1f/f-VcreERT2+: }}: 3, n_{\text {Btnl6f/f-VcreERT2+: }}: 3$, day 22: $n_{\text {ctrl: }}: 2, n_{\text {Btnl1f/f-VcreERT2+ }}: 1, n_{\text {Btnl6f/f-VcreERT2+ }}: 4$. c Top: Experimental scheme to analyse the effect of Btnl1 and Btnl6 tamoxifen-mediated depletion after 3 days. Bottom: $V \delta$-chain usage in $\vee \gamma 7^{+}$IEL in control (black), Btn/1/ff, Villin CreERT2+ (orange), Btnl6 $6^{\Delta g u t}$ (purple) and Btnl6f/f, VillinCreERT2+ (light blue) animals. The $V \delta 4$ and $V \delta 6.3$ chain gated on $V \gamma 7^{+}$IEL are plotted against each other. $\mathbf{d}$ Histogram of surface CD122 expression in indicated subpopulations of $V \gamma 7^{+} \mathrm{IEL}$ in animals of indicated genotypes. e Percentage of $\mathrm{V} \gamma 7$ cells (left) and usage of the $\mathrm{V} \delta 4$ and

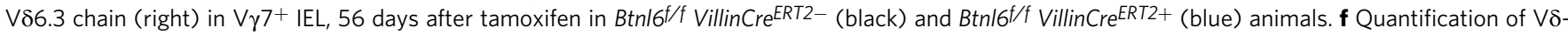
chain usage in $V \gamma 7^{+}$IEL in control, Btn/ $/ 7 / f$, Villin CreERT2+ and Btnl $6 / f$, Villin CreERT2+ knockout animals, 3 (left graph) and 56 days (right) after tamoxifen administration. Mean \pm SEM from two experiments per timepoint, left graph: $n_{\text {ctrl }}: 6, n_{\text {Btnllf/f-VcreERT2+ }}: 3, n_{\text {Btnll6f/f-VcreERT2+ }}: 6, n_{\text {Btnl6sgut: }}: 2$, right graph: $n_{\text {ctrl }}: 5$,

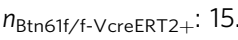

Because the murine gut epithelium expresses Btnl1, Btnl4 and Btnl6, it is also not obvious why Btnl1 + Btnl6 is the dominant selecting combination, although this might reflect expression levels (considered above), a prospect which cannot be investigated at the protein level until appropriate reagents are available.

Intriguingly, $\mathrm{V}_{\gamma} 7^{+}$IEL numbers did not decline over many weeks following acute Btnl gene locus ablation. This was surprising given that the IEL showed reduced expression of CD122, the receptor for IL-15 which is an important IEL growth factor ${ }^{47,48}$. Possibly, $\mathrm{V} \gamma 7^{+}$IEL were still able to compete for IL-15 because of the reduction in receptor expression by most such cells. Alternatively, the impact of reduced IL-15R expression on IEL might become evident by assessing the cells' replenishment in mice following infection or injury. The Btnl-dependence of sustained CD122 expression is also interesting in the light of reports that IL-15 regulates mucosal T-cell mobility within the gut, as part of immune surveillance $47,49,50$.

In this regard, an unanticipated observation was that although $\mathrm{V} 7^{+}$IEL numbers were maintained in mice acutely depleted of Btnl6, the TCR $\delta$ repertoire changed toward that seen in constitutive Btnl6 ${ }^{-/-}$mice. This presumably reflects ongoing replenishment of the gut IEL the half-lives of which have been reported to range from 2 to 14 weeks $^{51}$. We hypothesise that during a developmental window in early life, Btnl proteins are required to drive the selective differentiation and proliferation of IEL progenitors so that mature, expansive repertoires form. Thereafter, local self-renewal occurs from a mature progenitor pool, akin to that recently identified for memory CD8 T cells ${ }^{52}$, that does not require sustained Btnl expression but that is nonetheless influenced by it. Hence, somatic changes in Btnl expression patterns have the potential to change the IEL repertoire and status, as occurred in this study.

This scenario may model human disease settings where BTNL protein becomes altered, e.g. by inflammation or other gut pathophysiology ${ }^{24}$. However, the consequences may be greater than in mouse, because to date the potential to make only one type of heteromer $(\mathrm{BTNL} 3+8)$ has been identified in the human colon. Hence, the reduced expression of either BTNL protein, as has been reported in colon cancer (www.oncomine.org), might undermine the capacity to sustain the normal IEL repertoire and its functions in tissue maintenance, that have seemingly been conserved from agnathans to Homo sapiens. Finally, we note that future studies should investigate whether Skint/Btnl/BTNL heteromers exert cellautonomous effects on the epithelial cells that express them, outside of the impacts on their local lymphocyte compartments.

\section{Methods}

RNAscope. Rnascope was performed using probes for Btnll and Btnl6 according to the manufacturer's instructions. RNAscope was performed on paraffin embedded sections using probes and kits obtained from Advanced Cell Diagnostics/biotechne using the RNAscope 2.0 HD Reagent Kit-BROWN. Reference sequences are as follows: Btnl1, GenBank:NM_001111094.1 (576-1723); Btnl4, GenBank:NM_030746.1 (560-968); Btnl6, GenBank:NM_030747.1 (245-1552) and images were acquired using a Zeiss Axio/scan Z1 slide scanner and Zen Image acquisition software (Zen Blue, v2.6 Carl Zeiss Microscopy).

Tissue-specific deletion of genes. Tissue-specific deletion of genes was achieved by crossing floxed Cre-transgenic lines: Pgk-Cre (MGI: 2178050), Villin-Cre (MGI: 3053819) and VillinCre/ERT2 (MGI: 3053826). Tamoxifen (Sigma, T5648) dissolved in corn oil (Sigma, C8667) was administered on consecutive days as indicated via i.p. injection and animals were sacrificed on indicated timepoints. Successful deletion was confirmed by qPCR.

Spleen immunophenotyping. Comprehensive immunophenotyping of Btn6 $6^{-1-}$ mice was performed using a platform developed by the Wellcome Trust Infection and ImmunityImmunophenotyping (3i) consortium (www.immunophenotyping. org $)^{53}$. In brief, Spleen and MLN were digested with collagenase $(1 \mathrm{mg} / \mathrm{ml}) / \mathrm{DNAse}$ $(0.1 \mathrm{mg} / \mathrm{ml})$ in $2 \%$ FCS PBS $(+\mathrm{Ca} / \mathrm{Mg})$ for $20 \mathrm{~min}$ at $37^{\circ} \mathrm{C}$ and filtered through $30 \mu \mathrm{m}$ cell strainers. Cells were plated on 96-well V-bottom plates, washed in PBS and stained with Zombie Near-IR (Biolegend) for live/dead discrimination. Antibody stains were performed at $4{ }^{\circ} \mathrm{C}$ for $20 \mathrm{~min}$. Full details regarding phenotyping panels are included in Table S1. Samples were acquired on a BD LSR Fortessa X-20 equipped with $405 \mathrm{~nm}(40 \mathrm{~mW}), 488 \mathrm{~nm}(50 \mathrm{~mW}), 561 \mathrm{~nm}(50 \mathrm{~mW})$ and $640 \mathrm{~nm}$ $(100 \mathrm{~mW})$ lasers.

Mice. Wild-type (WT) C57BL/6J and FVB mice were obtained from Jackson Laboratories. NF-Skint1 ${ }^{\mathrm{Tg}}$, Btnl1-KOMP, Btnl4-KOMP and Skint1 ${ }^{\Delta / \Delta}$ mice have been previously described ${ }^{16,29,32}$. Genetically engineered mice were generated at the Francis Crick Institute's Transgenic Facility. The sg RNAs \& PAM sequences (see Table 1) were cloned into the g-RNA basic vector, translated in vitro, purified and co-injected with Cas 9 into day 1 zygotes and transferred into pseudopregnant foster mice by the Francis Crick Institute's Transgenic Facility. Targeted animals were identified and validated by PCR and later genotyped using the Transnetyx platform. All animals were maintained at The Francis Crick Institute's Biological resource facilities with a $12 \mathrm{~h}$ light/dark cycle and access to food and water ad libitum, temperature $19-23^{\circ} \mathrm{C}, 55 \pm 10 \%$ humidity. Animal experiments were undertaken in full compliance with UK Home Office regulations and under a project license to A.C.H. (7009056).

Generation of Skint1, Skint2 rat monoclonal antibodies. Rat monoclonal antibodies against Skint1 and Skint2 were generated by immunization of Lou/c rats with purified GST-tagged human Skint1 or Skint2 extracellular domain, respectively. Hybridoma cells were generated and binding to Skint1 or Skint2 protein was analysed by enzyme-linked immunosorbent assay (ELISA). Positive hybridoma supernatants were further assayed for their potential in immunoblotting. Hybridoma clones Skint $12 \mathrm{G} 2$ and Skint2 3G8 (both IgG2a/k) were recloned by limiting dilution to obtain stable monoclonal cell lines.

Quantitative RT-PCR. Samples were stored in RNAlater (Ambion) or directly frozen in RLT buffer prior to RNA purification with DNAse digest (QIAGEN RNeasy kit). cDNA was generated using Superscript-II (Invitrogen) and analysed using Sybr-green assay (Invitrogen) using a Quant-studio 5 or Viaa7 Real-time PCR machine (Applied Biosystems) and qPCR primers indicated in Table 2.

Isolation of mouse intestinal IEL. Mouse IEL were isolated from small intestine ${ }^{18}$ Briefly, small intestine was opened, washed in PBS, cut into $0.5-\mathrm{cm}$ long pieces and 
a

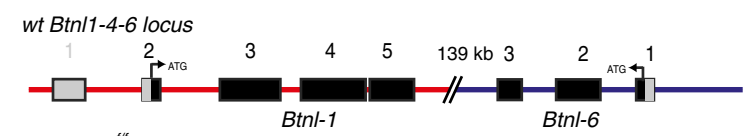

Btnl 1-4-6 6 f/f locus
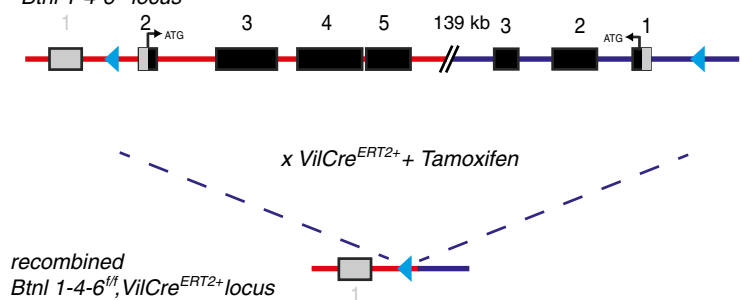

Btn1146ff/,VillinCreERT2

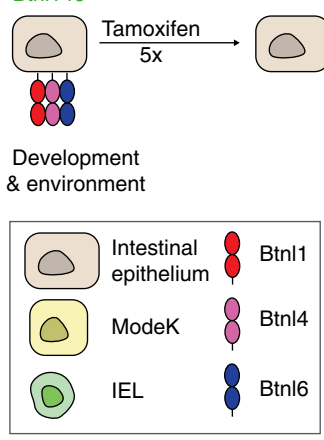

54 days
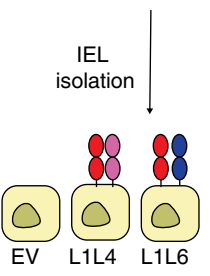

Co-culture with ModeK b

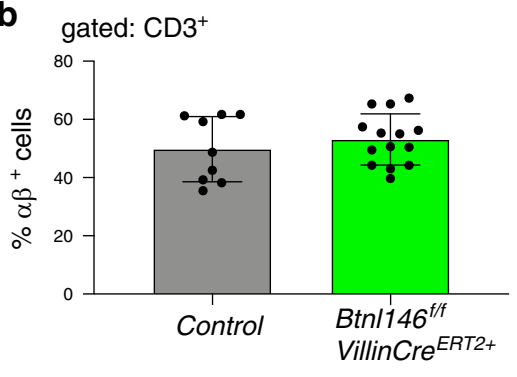

$d+54$

C

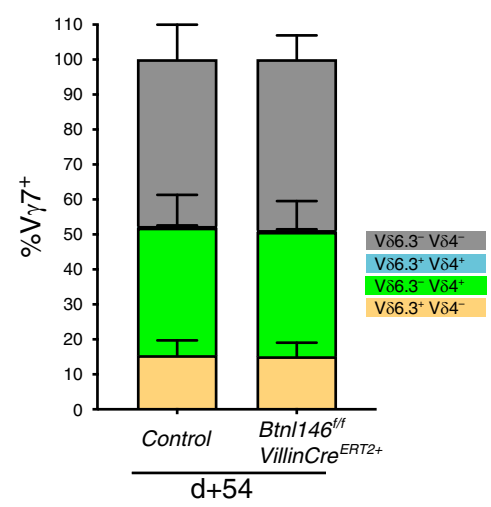

e

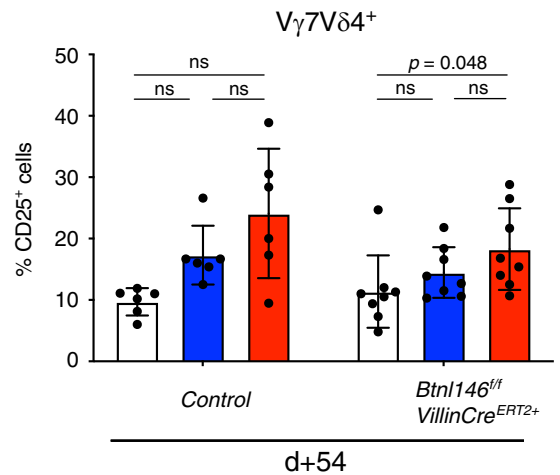

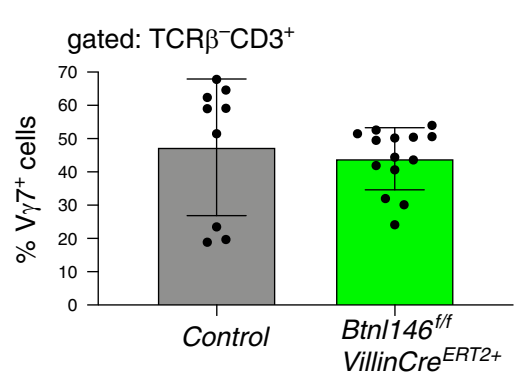

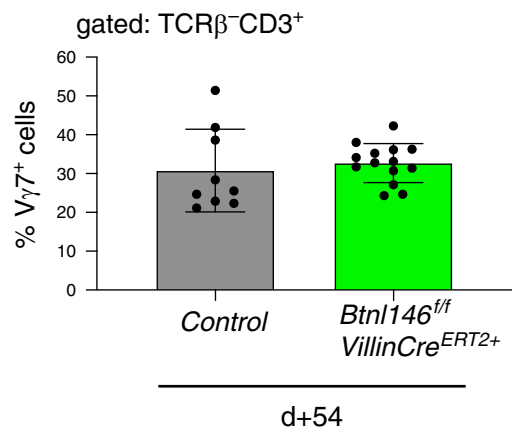

$d+54$ d
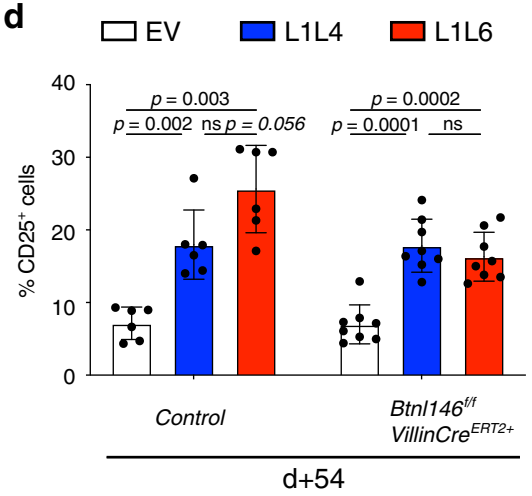

$\mathrm{V} \gamma 7 \mathrm{~V} \delta 6.3^{+}$

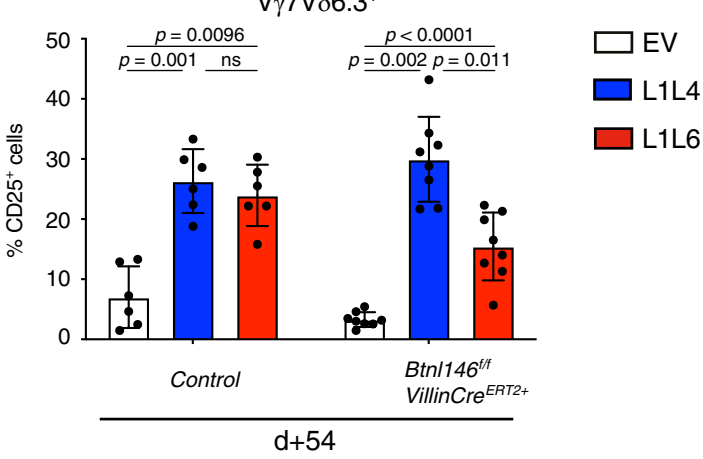

Fig. 6 Response and maintenance by Btnl1 and Btnl6. a Left: Targeting strategy to generate animals harbouring a floxed Btnl146 locus, which can be excised after tamoxifen administration. Right: Experimental design for IEL analysis (c, d) and co-culture experiment (e) following Btnl146 locus depletion. During development Btnl molecules are expressed on the IEC and only after tamoxifen depletion Btnl expression is lost. Following loss of Btnl expression, IELs are harvested and subjected to co-cultures with MODE-K cells expressing specific Btnl combination. $\mathbf{b}$ Quantification of $\alpha \beta$ (left), $V \gamma 7^{+}$(middle) and

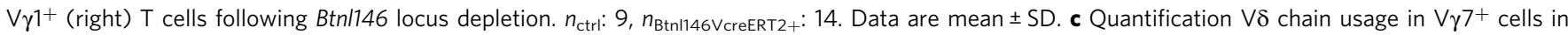
animals of indicated genotypes under indicated conditions. $n_{\text {ctrl }}: 6, n_{\text {Btnl146VcreERT2+ }}: 8$. Data are mean \pm SD. $\mathbf{d}$ Co-culture of MODE-K cells transduced with EV, L1L4 or L16 with IEL from control, or Btn/146f/f; Villin CreERT2+ animals. Controls are pooled: Btn/146f/f, Villin CreERT2+ that did not receive tamoxifen and Btnl146/f, VillinCreERT2- animals that did receive Tamoxifen. Data are mean \pm SD $\left(n_{\text {ctrl }}: 6, n_{\text {Btnl146VcreERT2+ }}: 8\right)$. Statistical analysis two-way ANOVA \& Tukey's multiple comparison post test. e IEL response in co-cultures of MODE-K cells transduced with EV, L1L4 or L1L6 with IEL from control or Btn/146f/f; Villin CreERT2+ animals that did receive tamoxifen in $\mathrm{V} 7 \mathrm{~V} \delta 4^{+}$(left graph) and $\vee \gamma 7 \mathrm{~V} 86.3^{+}$(right graph) cells. $n_{\text {ctrll }}: 6, n_{\text {Btnl1446VcreERT2+ }}: 8$. 


\section{Table 1 Oligos and repair templates used for generation of} floxed mice.

\section{Short guide oligo (sg) and Sequence 5'-3 homology repair (HDR) \\ Btnl6-5'sg-2 \\ Btnl6-3'sg-2 \\ Btnl6-5' HDR template \\ Btnl6-3'HDR template \\ Btnl1 Int12 sg-3 \\ Btnl1 Int56 sg-2 \\ Btnl1-HDR template_1 \\ Btnl1-HDR template_2 \\ TAACCTGGGGAGGAGTTAAGAGG \\ AGGATTCACACTGACAACTTAGG \\ AGCAGAGATGGCTTGCGGTGATTTTC \\ CATGTCCAGCAGAACTGAAGAGAAAAA \\ CAGGAGAGGCAGATCAATAACCTGGGTA \\ CCATAACTTCGTATAGCATACATTATACGA \\ AGGTATGGGAGGAGTTAAGAGACCAAAT \\ CCACCCAGATCTTGGACCCCTCCTCAGA \\ GACAGCATTGC \\ AGGCTCCAGGCCCTTCCAGGACCCAT \\ GGGGGCTTTGGCCTGTGGCTTCTACAC \\ TACTACAAGGATTCACACTGACAAGGT \\ ACCATAACTTCGTATAGCATACATTATACG \\ AAGTTATCTTAGGCAGTGGTCCAGACTAT \\ GGGAACAGAGAGTTCCTGGCATGCTGG \\ AGGAATGGAGAGTCTTC \\ CCCAAGGGGGATCTTGGAGCTGG \\ TCCATAGCACCTTATCCGGTTGG \\ AATGTGGGAGTGGTCTACTTTCTTGTAT \\ GACTTCACTGCCCTACATTGGACTCAG \\ AGAACCCAGCTTAATTAAATAACTTCGT \\ ATAGCATACATTATACGAAGTTATCCAAG \\ ATCCCCCTTGGGACCATGAACTCACAG \\ AAAGGCGAGAGAAAATGGGAACTTGGC \\ AGCTTTCCATGTCCACGG \\ AAGCCCTAAGACACCTTAAACTCCCAA \\ GGTGCTGGGACATTGCTCTGTGACTCC \\ ATAGCACCTTATTAATTAAATAACTTCG \\ TATAGCATACATTATACGAAGTTATCCGG \\ TTGGTGTCCCTGTGAGCATGCTCATCTC \\ CTTTATCATGGGGCCTCTACGGGAACGC \\ CAAGTCTAATTCGTTAG}

Bold letters indicate the PAM, underlined letters indicate the restriction site and italic letters indicate loxP.

incubated at RT on awheel in complete RPMI supplemented with $1 \mathrm{mM}$ DTT. Tissues were then washed, vortexed in complete RPMI and filtered through $70 \mathrm{~nm}$ nylon cell strainers. Vortexing and filtration steps were repeated twice. IEL were then purified by Percoll density centrifugation and stained by flow cytometry (for antibodies, see Table 3).

IEL cultures. IEL cultures were performed ${ }^{19}$. Briefly, $10^{5}$ MODE-K cells were plated onto 48 -well plates $24 \mathrm{~h}$ prior to co-culture experiments. The following day, the medium was removed and $10^{5}$ unsorted IEL suspended in $200 \mu$ of RPMI 1640 supplemented with L-glutamine, $10 \%$ heat-inactivated FCS, $1 \%$ pen/strep, 10 $\mathrm{mM}$ hepes, $1 \mathrm{mM}$ sodium pyruvate, $1 \mathrm{x}$ non-essential amino acids, $50 \mu \mathrm{M} \beta$-mercaptoethanol, IL-2 $10 \mathrm{U} / \mathrm{ml}$, IL-15 $10 \mathrm{ng} / \mathrm{ml}$ (Immunotools), IL-3 $100 \mathrm{U} / \mathrm{ml}$ and IL$4200 \mathrm{U} / \mathrm{ml}(\mathrm{R} \& \mathrm{D})$ were seeded on top of the monolayer. Cells were co-cultured overnight $(16-18 \mathrm{~h})$ at $37^{\circ} \mathrm{C}$ with $10 \% \mathrm{CO}_{2}$.

Flow Cytometry acquisition was performed using BD-FACS/Diva Software. Data analysis was performed using FlowJo v10 10.6.1 (FlowJo, LLC, Ashland OR).

Cell lines. HEK293T cells (FCI) were maintained in DMEM supplemented with $4.5 \mathrm{~g} / \mathrm{l} \mathrm{d}$-glucose, l-glutamine, $10 \%$ heat-inactivated FCS and $1 \%$ penicillin streptomycin (complete DMEM). Transgenic MODE-K cell lines ${ }^{19}$ were maintained in complete DMEM supplemented with $1 \mu \mathrm{g} / \mathrm{ml}$ puromycin (Sigma-Aldrich) and $500 \mu \mathrm{g} / \mathrm{ml}$ hygromycin (Thermo Fisher). Transgenic J76 cells ${ }^{19}$ were maintained in RPMI 1640 l-glutamine, 10\% FCS and 1\% penicillin-streptomycin. All cell culture reagents were from Thermo Fisher.

Cell line co-culture. In all, $5 \times 10^{4}$ transduced J76 was mixed in 96-well plates with $2 \times 10^{5}$ transiently transfected $293 \mathrm{~T}$ cells, followed by co-culture for $5 \mathrm{~h}$.

Plasmids and transfection. Overlap-extension PCR (OE-PCR) was used to replace the GFG regions of Btnl4 with those of Btnll on plasmids encoding Btnl1/ $4 / 6^{16}$. HEK293T cells were transfected with the indicated combinations of FLAGBtnl1, HA-Btnl6, HIS-Btnl4 and empty vector (EV) encoding plasmids. Medium was replaced $16 \mathrm{~h}$ after transfection and cells were harvested at $48 \mathrm{~h}$ and used for the co-culture assay. For antibodies see Table 3.

Preparation of epidermal sheets. Ear epidermis was separated from dermis following incubation in $0.5 \mathrm{M}$ ammonium thiocyanate for $35 \mathrm{~min}$ at $37^{\circ} \mathrm{C}$. Isolated
Table 2 qPCR primers.

\begin{tabular}{lll} 
Target & Forward & Reverse \\
\hline Mu-Btnl-1 & TGACCAGGAGAAATCGAAGG & CACCGAGCAGGACCAATAGT \\
Mu-Btnl-4 & CATTCTCCTCAGAGACCCACACTA & GAGAGGCCTGAGGGAGAA \\
Mu-Btnl-6 & GCACCTCTCTGGTGAAGGAG & ACCGTCTTCTGGACCTTTGA \\
Mu-Ppia & CAAATGCTGGACCAAACACAA & CCATCCAGCCATTCAGTCTTG \\
Mu-Skint1 & AAACAAAAGGGAGCTGACCC & CCCCTCTAAGCCGTTCACTA \\
Mu-Skint2 & GCTACAGGAGTACTTCTCTGTGTTGT & TGGTGCCAAGACTGGCCT \\
Mu-Psmb9 & GTCGTGGTGGGCTCTGATT & GAACCTGAGAGGGCACAGAA \\
Mu-Btnl2 & TTTGCTATGGATGACCCTGC & TCCTGATTGCTGCTGTGTGT \\
\hline
\end{tabular}

epidermal sheets were fixed with ice cold acetone at $-20^{\circ} \mathrm{C}$. The samples were blocked in 5\% FCS for $1 \mathrm{~h}$ at room temperature and stained for $1 \mathrm{~h}$ at $37^{\circ} \mathrm{C}$ using $\mathrm{V} \gamma 3$ TCR-FITC (clone 536, BD), MHC I-A/I-E-AF647 (clone M5/114.15.2, BioLegend) and CD45-eFluor450 (clone 30-F11, eBioscience) antibodies. Tissue samples were mounted on microscope glass in Prolong Gold mounting medium under a stereomicroscope to ensure flat epidermal mounting. Confocal images were recorded using Leica SP5 confocal microscope with 40× 1.25 NA HCX PL APO CS lens. Three confocal records $387.5 \times 387.5 \mu \mathrm{m}$ size were acquired from each epidermal sheet. Image quantification was performed using Definiens Developer software (version XD2.7). Each channel in a record was processed with Gaussian filter followed by application of automated multi-threshold segmentation. Individual cells (CD45+, Langerhans cells, and T cells) were detected based on their relative intensity in CD45, MHC II and TCR channels, respectively. Cell number and morphology were measured for each cell type.

Preparation of lung and uterus $\boldsymbol{\gamma} \boldsymbol{\delta}$ cells. Lungs and uteri from experimental mice were collected in medium and minced with razor blades. Samples were digested using Miltenyi Multi Tissue Dissociator kit 1, according to the manufacturer's instructions. Briefly, samples were transferred to GentleMACS C tubes containing $2.5 \mathrm{~mL}$ digestion mix (100 $\mu \mathrm{L}$ Enzyme D, $50 \mu \mathrm{L}$ Enzyme $\mathrm{R}$ and $12.5 \mu \mathrm{L}$ Enzyme A) and incubated at $37^{\circ} \mathrm{C}$ for $40 \mathrm{~min}$ with shaking. Following incubation, single-cell suspensions were prepared by homogenisation using GentleMACS program C and filtering through $70 \mu \mathrm{m}$ cell strainers. Single-cell suspensions were stained with Live/Dead Aqua for dead cell exclusion, followed by Fc-block and surface stain with specific antibodies.

Biochemistry. Cells were lysed for $30 \mathrm{~min}$ in ice cold RIPA buffer with protease inhibitors (Roche) and phosphatase inhibitors (Phosphatase inhibitor cocktails 2 \& 3 , Sigma) and spun at $20,000 \times g$ for $15 \mathrm{~min}$ at $4{ }^{\circ} \mathrm{C}$. Protein concentrations in supernatants was determined using a BCA kit (Pierce).

Immunoprecipitation from cell lysates. Lysates were precleared on Protein G Sepharose (Millipore-Sigma) for $1 \mathrm{~h}$, incubated with antibodies for $1 \mathrm{~h}$ followed by incubation with $1 \%$ BSA blocked Protein G beads for a further hour. Following three washes in RIPA buffer immunoprecipates or samples were mixed with NuPAGE LDS Sample Buffer supplemented with 1x NuPAGE reducing agent and separated by electrophoresis on NuPage $4-12 \%$ Bis-Tris protein gels (Thermo Fisher) and then transferred onto PVDF membranes. Membranes were blocked in PBS- $0.1 \%$ Tween 20 and 5\% BSA for $60 \mathrm{~min}$ at room temperature and incubated with primary antibodies overnight. Membranes were washed three times with PBS$0.1 \%$ Tween 20 and incubated for 60 min with HRP conjugated secondary antibodies, washed again and developed using ECL detection reagents (Merck).

Immunoprecipitation from thymus. 12 Thymi of E17/E18 FVB and 22 Thymi of NF-Skint $1^{T g}$ pups were lysed as described above and incubated with Flag-M2 coated beads overnight at $4{ }^{\circ} \mathrm{C}$. Following three washes in RIPA buffer immunoprecipates were eluted with $3 x$ Flag peptide to obtain the eluate or beads were directly boiled for analysis. Beads, Eluate or samples were mixed with NuPAGE LDS Sample Buffer supplemented with 1x NuPAGE reducing agent and separated by electrophoresis on NuPage $4-12 \%$ Bis-Tris protein gels (Thermo Fisher) and then transferred onto PVDF membranes. Membranes were blocked in PBS- $0.1 \%$ Tween 20 and $5 \%$ BSA for $60 \mathrm{~min}$ at room temperature and incubated with primary antibodies overnight. Membranes were washed three times with PBS- $0.1 \%$ Tween 20 and incubated for 60 min with HRP conjugated secondary antibodies, washed again and developed using ECL detection reagents (Merck).

Bioinformatics analysis. Raw gene counts were obtained from GSE109413 (Moor et al.) ${ }^{54}$ and GSE92332 (Haber et al.) ${ }^{55}$ and each cell-set was preprocessed using Seurat ${ }^{56}$ (version 3.1.1.9023). In the case of data from Moor et al. data, cells with $<200$ and $>3000$ detectable genes and cells with percentage mitochondrial expression greater $5 \%$ were removed. For all cell-sets, the total counts were scaled to $1 \mathrm{e} 4$ counts, a log transformation applied and genes were $\mathrm{z}$-score across all cells. In the case of data from Moor et al., both replicate cell-sets were merged using Seurat's IntegrateData function after which the gene-wise vectors were rescaled. The dot plots were produced using the DotPlot function from Seurat and profile plots were produced across the villus regions using the $\mathrm{z}$-score scaled data. 
Table 3 Antibodies used for flow cytometry, western blotting and microscopy.

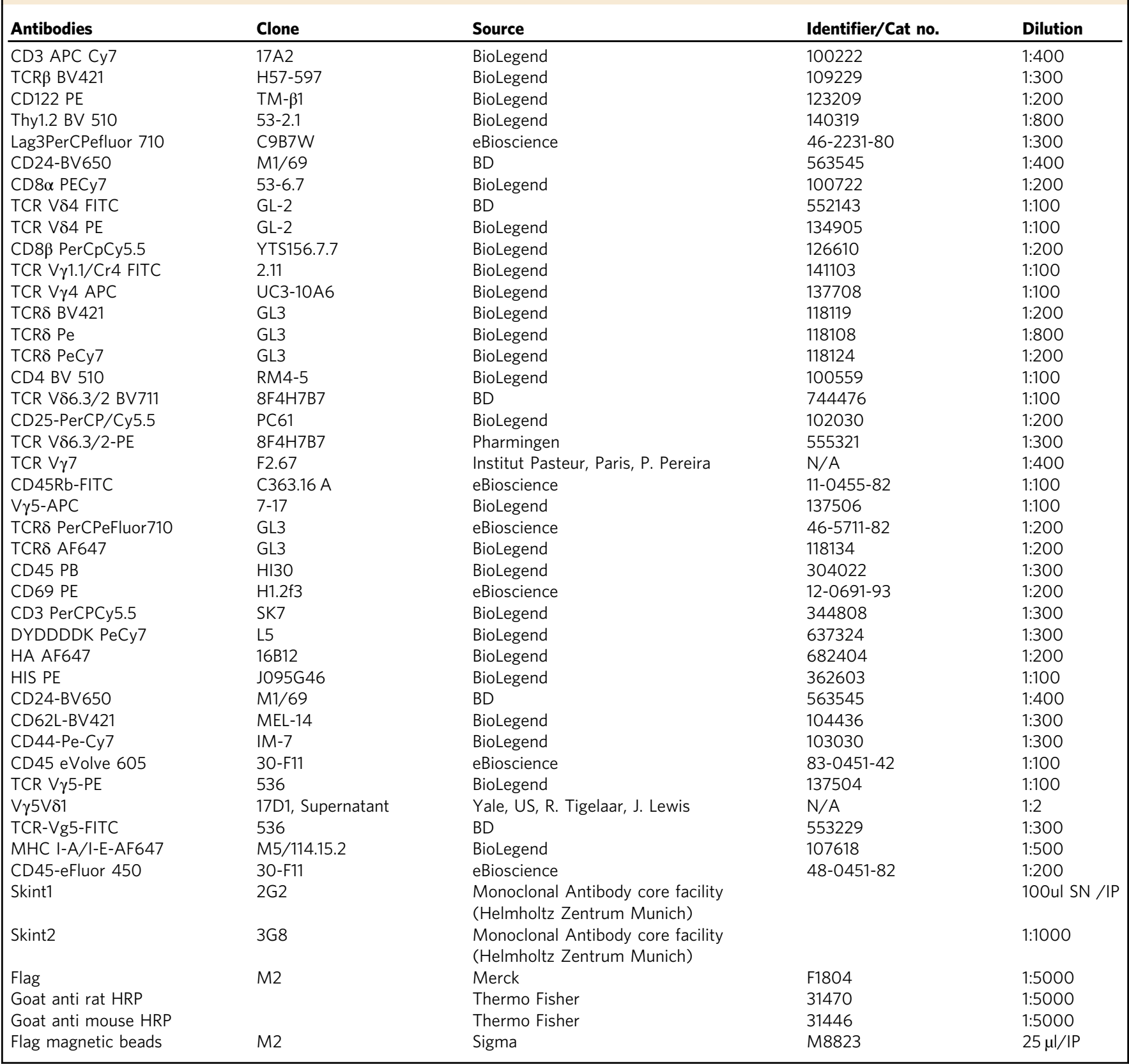

Statistical analysis. Summary data are represented as mean \pm SD if representative experiments are shown or mean \pm SEM if summarized data are shown as indicated in individual figures. Numbers of animals per group are indicated in individual figures.

Control groups includes animals that are wt, heterozygous or homozygous without the respective Cre transgene. Heterozygous animals are comparable to WT animals.

Modelling software. Figures for all modelling data were generated in PyMOL v2.0.7 (Schrodinger LLC). 3D-JIGSAW was used to generate 3D models of proteins and perform docking simulations, respectively.

Reporting summary. Further information on research design is available in the Nature Research Reporting Summary linked to this article.

\section{Data availability}

This work did not include any data which mandated deposition in public databases. Associated raw data are provided in the main and/or supplementary figures. Relations to summary data charts are indicated and a full list of figures with associated raw data is provided in the reporting summary linked to this article. Raw gene counts were obtained from GSE109413 (Moor et al.) 54 and GSE92332 (Haber et al.) ${ }^{55}$. For bioinformatics single-cell analysis scripts are available on github: https://github.com/ajandke/ Jandke_etal_naturecomms. Immunophenotyping data for pipeline procedure can be found under https://www.mousephenotype.org/data/secondaryproject/3i.

\section{Code availability}

The full source codes have not been released. Publicly available servers can be accessed online for 3D-JIGSAW (https://bmm.crick.ac.uk/ svc-bmm-3djigsaw/). For bioinformatics single-cell analysis, scripts are available on github: https://github.com/ ajandke/Jandke_etal_naturecomms.

Received: 5 November 2019; Accepted: 26 June 2020; Published online: 28 July 2020

\section{References}

1. Zhu, J. et al. Immune surveillance by CD8aa+ skin-resident $\mathrm{T}$ cells in human herpes virus infection. Nature 497, 494-497 (2013). 
2. Perdiguero, E. G. \& Geissmann, F. The development and maintenance of resident macrophages. Nat. Immunol. 17, 2-8 (2016).

3. Panduro, M., Benoist, C. \& Mathis, D. Tissue Tregs. Annu Rev. Immunol. 34, 609-633 (2016).

4. Nielsen, M. M., Witherden, D. A. \& Havran, W. L. $\gamma \delta$ T cells in homeostasis and host defence of epithelial barrier tissues. Nat. Rev. Immunol. 17, 733-745 (2017).

5. Goodman, T. \& Lefrancois, L. Intraepithelial lymphocytes. Anatomical site, not $\mathrm{T}$ cell receptor form, dictates phenotype and function. J. Exp. Med. 170, 1569-1581 (1989).

6. Goodman, T. \& Lefrancois, L. Expression of the gamma-delta T-cell receptor on intestinal CD8 + intraepithelial lymphocytes. Nature 333, 855-858 (1988).

7. Havran, W. L. \& Allison, J. P. Origin of Thy-1+ dendritic epidermal cells of adult mice from fetal thymic precursors. Nature 344, 68-70 (1990).

8. Kyes, S., Pao, W. \& Hayday, A. Influence of site of expression on the fetal gamma delta T-cell receptor repertoire. Proc. Natl Acad. Sci. USA $\mathbf{8 8}$ 7830-7833 (1991).

9. Girardi, M. et al. Regulation of cutaneous malignancy by gammadelta T cells. Science 294, 605-609 (2001).

10. Hayday, A. \& Tigelaar, R. Immunoregulation in the tissues by gammadelta T cells. Nat. Rev. Immunol. 3, 233-242 (2003).

11. Born, W. et al. Immunoregulatory functions of $\gamma \delta \mathrm{T}$ cells. Adv. Immunol. 71, 77-144 (1999).

12. Jameson, J. et al. A role for skin $\gamma \delta \mathrm{T}$ cells in wound repair. Science 296, 747-749 (2002).

13. Hayday, A. C. $\gamma \delta$ cells: a right time and a right place for a conserved third way of protection. Annu Rev. Immunol. 18, 975-1026 (2000).

14. Strid, J. et al. Acute upregulation of an NKG2D ligand promotes rapid reorganization of a local immune compartment with pleiotropic effects on carcinogenesis. Nat. Immunol. 9, 146-154 (2008).

15. Hayday, A. C. $\gamma \delta$ T cells and the lymphoid stress-surveillance response. Immunity 31, 184-196 (2009).

16. Di Marco Barros, R. et al. Epithelia use butyrophilin-like molecules to shape organ-specific $\gamma \delta$ T cell compartments. Cell 167, 203-218 e217 (2016).

17. Boyden, L. M. et al. Skint1, the prototype of a newly identified immunoglobulin superfamily gene cluster, positively selects epidermal $\gamma \delta$ T cells. Nat. Genet 40, 656-662 (2008).

18. Turchinovich, G. \& Hayday, A. C. Skint-1 identifies a common molecular mechanism for the development of interferon- $\gamma$-secreting versus interleukin17-secreting $\gamma \delta$ T cells. Immunity 35, 59-68 (2011).

19. Melandri, D. et al. The gammadeltaTCR combines innate immunity with adaptive immunity by utilizing spatially distinct regions for agonist selection and antigen responsiveness. Nat. Immunol. 19, 1352-1365 (2018).

20. Rigau, M. et al. Butyrophilin $2 \mathrm{~A} 1$ is essential for phosphoantigen reactivity by $\gamma \delta$ T cells. Science https://doi.org/10.1126/science.aay5516 (2020).

21. Harly, C. et al. Key implication of CD277/butyrophilin-3 (BTN3A) in cellular stress sensing by a major human $\gamma \delta$ T-cell subset. Blood 120, 2269-2279 (2012).

22. Wang, $H$. et al. Butyrophilin $3 \mathrm{~A} 1$ plays an essential role in prenyl pyrophosphate stimulation of human $\mathrm{V} \gamma 2 \mathrm{~V} \delta 2 \mathrm{~T}$ cells. J. Immunol. 191, 1029-1042 (2013).

23. $\mathrm{Gu}, \mathrm{S}$. et al. Phosphoantigen-induced conformational change of butyrophilin $3 \mathrm{~A} 1$ (BTN3A1) and its implication on V $\gamma 9 \mathrm{~V} \delta 2 \mathrm{~T}$ cell activation. Proc. Natl Acad. Sci. USA 114, E7311-E7320 (2017).

24. Mayassi, T. et al. Chronic inflammation permanently reshapes tissue-resident immunity in celiac disease. Cell https://doi.org/10.1016/j.cell.2018.12.039 (2019).

25. Vantourout, P. et al. Heteromeric interactions regulate butyrophilin (BTN) and BTN-like molecules governing $\gamma \delta$ T cell biology. Proc. Natl Acad. Sci. USA 115, 1039-1044 (2018).

26. Yang, Y. et al. A structural change in butyrophilin upon phosphoantigen binding underlies phosphoantigen-mediated $\mathrm{V} \gamma 9 \mathrm{~V} \delta 2 \mathrm{~T}$ cell activation. Immunity https://doi.org/10.1016/j.immuni.2019.02.016 (2019).

27. Palakodeti, A. et al. The molecular basis for modulation of human $\mathrm{V} \gamma 9 \mathrm{~V} \delta 2 \mathrm{~T}$ cell responses by CD277/butyrophilin-3 (BTN3A)-specific antibodies. J. Biol. Chem. 287, 32780-32790 (2012)

28. Sandstrom, A. et al. The intracellular B30.2 domain of butyrophilin $3 \mathrm{~A} 1$ binds phosphoantigens to mediate activation of human $\mathrm{V} \gamma 9 \mathrm{~V} \delta 2 \mathrm{~T}$ cells. Immunity 40, 490-500 (2014).

29. Narita, T., Nitta, T., Nitta, S., Okamura, T. \& Takayanagi, H. Mice lacking all of the Skint family genes. Int. Immunol. https://doi.org/10.1093/intimm/ dxy030 (2018).

30. Lewis, J. M. et al. Selection of the cutaneous intraepithelial $\gamma \delta+$ T cell repertoire by a thymic stromal determinant. Nat. Immunol. 7, 843-850 (2006).

31. Mohamed, R. H. et al. The SKINT1-like gene is inactivated in hominoids but not in all primate species: implications for the origin of dendritic epidermal T cells. PLoS ONE 10, e0123258, https://doi.org/10.1371/journal.pone.0123258 (2015).
32. Barbee, S. D. et al. Skint-1 is a highly specific, unique selecting component for epidermal T cells. Proc. Natl Acad. Sci. USA 108, 3330-3335 (2011)

33. Bas, A. et al. Butyrophilin-like 1 encodes an enterocyte protein that selectively regulates functional interactions with T lymphocytes. Proc. Natl Acad. Sci. USA 108, 4376-4381 (2011)

34. Lebrero-Fernandez, C., Bergstrom, J. H., Pelaseyed, T. \& Bas-Forsberg, A. Murine butyrophilin-like 1 and Btnl6 form heteromeric complexes in small intestinal epithelial cells and promote proliferation of local T lymphocytes. Front. Immunol. 7, 1 (2016).

35. Lallemand, Y., Luria, V., Haffner-Krausz, R. \& Lonai, P. Maternally expressed PGK-Cre transgene as a tool for early and uniform activation of the Cre sitespecific recombinase. Transgenic Res. 7, 105-112 (1998).

36. el Marjou, F. et al. Tissue-specific and inducible Cre-mediated recombination in the gut epithelium. Genesis 39, 186-193 (2004).

37. Willcox, C. R. et al. Butyrophilin-like 3 directly binds a human $\mathrm{V} \gamma 4+\mathrm{T}$ cell receptor using a modality distinct from clonally-restricted antigen. Immunity, https://doi.org/10.1016/j.immuni.2019.09.006 (2019).

38. Lebrero-Fernandez, C. et al. Altered expression of Butyrophilin (BTN) and BTN-like (BTNL) genes in intestinal inflammation and colon cancer. Immun. Inflamm. Dis. 4, 191-200 (2016).

39. Hoytema van Konijnenburg, D. P. et al. Intestinal epithelial and intraepithelial $\mathrm{T}$ cell crosstalk mediates a dynamic response to infection. Cell 171, 783-794 e713 (2017).

40. Kohlgruber, A. C. et al. $\gamma \delta \mathrm{T}$ cells producing interleukin-17A regulate adipose regulatory $\mathrm{T}$ cell homeostasis and thermogenesis. Nat. Immunol. 19, 464-474 (2018).

41. Edelblum, K. L. et al. $\gamma \delta$ intraepithelial lymphocyte migration limits transepithelial pathogen invasion and systemic disease in mice. Gastroenterology 148, 1417-1426 (2015)

42. He, S. et al. Gut intraepithelial $\mathrm{T}$ cells calibrate metabolism and accelerate cardiovascular disease. Nature 566, 115-119 (2019).

43. Vantourout, P. \& Hayday, A. Six-of-the-best: unique contributions of gammadelta T cells to immunology. Nat. Rev. Immunol. 13, 88-100 (2013).

44. Wu, Y. et al. An innate-like $V \delta 1^{+} \gamma \delta \mathrm{T}$ cell compartment in the human breas is associated with remission in triple-negative breast cancer. Sci Transl Med 11, https://doi.org/10.1126/scitranslmed.aax9364 (2019).

45. Karunakaran, M. M. et al. Butyrophilin-2A1 directly binds germline-encoded regions of the $\mathrm{V} \gamma 9 \mathrm{~V} \delta 2 \mathrm{TCR}$ and is essential for phosphoantigen sensing. Immunity, https://doi.org/10.1016/j.immuni.2020.02.014 (2020).

46. Kreslavsky, T. et al. TCR-inducible PLZF transcription factor required for innate phenotype of a subset of $\gamma \delta$ T cells with restricted TCR diversity. Proc. Natl Acad. Sci. USA 106, 12453-12458 (2009).

47. $\mathrm{Hu}, \mathrm{M}$. D. et al. Epithelial IL-15 is a critical regulator of $\gamma \delta$ intraepithelial lymphocyte motility within the intestinal mucosa. J. Immunol. https://doi.org/ 10.4049/jimmunol.1701603 (2018).

48. Lai, Y. G. et al. IL-15 does not affect IEL development in the thymus but regulates homeostasis of putative precursors and mature CD8 + IELs in the intestine. J. Immunol. 180, 3757-3765 (2008).

49. Maiuri, L. et al. IL-15 drives the specific migration of CD94+ and TCR- $\gamma \delta+$ intraepithelial lymphocytes in organ cultures of treated celiac patients. Am. J. Gastroenterol. 96, 150-156 (2001)

50. Sowell, R. T. et al. IL-15 complexes induce migration of resting memory CD8 T cells into mucosal tissues. J. Immunol. 199, 2536-2546 (2017).

51. Penney, L., Kilshaw, P. J. \& MacDonald, T. T. Regional variation in the proliferative rate and lifespan of alpha beta TCR + and $\gamma \delta$ TCR + intraepithelial lymphocytes in the murine small intestine. Immunology 86, 212-218 (1995).

52. Im, S. J. et al. Defining CD8 + T cells that provide the proliferative burst after PD-1 therapy. Nature 537, 417-421 (2016).

53. Abeler-Dorner, L. et al. High-throughput phenotyping reveals expansive genetic and structural underpinnings of immune variation. Nat. Immunol. 21, 86-100 (2020).

54. Moor, A. E. et al. Spatial reconstruction of single enterocytes uncovers broad zonation along the intestinal villus axis. Cell 175, 1156-1167 e1115 (2018)

55. Haber, A. L. et al. A single-cell survey of the small intestinal epithelium. Nature 551, 333-339 (2017).

56. Butler, A., Hoffman, P., Smibert, P., Papalexi, E. \& Satija, R. Integrating singlecell transcriptomic data across different conditions, technologies, and species. Nat. Biotechnol. 36, 411-420 (2018).

\section{Acknowledgements}

We thank Professor D. Kaiserlian (INSERM U1111, Lyon) for MODE-K cells; Dr P. Pereira (Institut Pasteur) for the hybridoma (F2.67) producing antibody to TCR V $\gamma 7+$ TCR; and Drs Raphaël A. G. Chaleil and Paul Bates in the Biomolecular Modelling Laboratory of The Francis Crick Institute, London, UK. We are grateful to the flow cytometry, genomic equipment park, bioinformatics, experimental histopathology, proteomics platform, cell services, and biological service units of the Francis Crick Institute 
and to scientific facilities support at King's College London. This work was supported by a Wellcome Trust Investigator Award, 106292/Z14/Z and the Francis Crick Institute which receives its core funding from Cancer Research UK (FC001093), the UK Medical Research Council (FC001093), and the Wellcome Trust (FC001093). L.M. was supported by the European Union's Horizon 2020 research and innovation programme under the Marie Skłodowska-Curie grant agreement No. 792383. D.M. received studentships from the King's Bioscience Institute and the Guy's and St. Thomas' Charity Prize PhD program in Biomedical and Translational Science.

\section{Author contributions}

A.J., D.M., L.M., D.U., A.L. and P.V. designed and undertook experiments; P.E. performed bioinformatics analysis on public datasets; T.Ni., T.Na. and H.T. provided Skint1-ko mice; R.F. generated antibodies; A.J. and A.H. designed the study and wrote the paper.

\section{Competing interests}

A.C.H. is equity holder in GammaDelta Therapeutics and in Adaptate Biotherapeutics. The remaining authors declare no competing interests.

\section{Additional information}

Supplementary information is available for this paper at https://doi.org/10.1038/s41467020-17557-y.

Correspondence and requests for materials should be addressed to A.H.
Peer review information Nature Communications thanks the anonymous reviewer(s) for their contribution to the peer review of this work.

Reprints and permission information is available at http://www.nature.com/reprints

Publisher's note Springer Nature remains neutral with regard to jurisdictional claims in published maps and institutional affiliations.

(c) (i)

Open Access This article is licensed under a Creative Commons Attribution 4.0 International License, which permits use, sharing, adaptation, distribution and reproduction in any medium or format, as long as you give appropriate credit to the original author(s) and the source, provide a link to the Creative Commons license, and indicate if changes were made. The images or other third party material in this article are included in the article's Creative Commons license, unless indicated otherwise in a credit line to the material. If material is not included in the article's Creative Commons license and your intended use is not permitted by statutory regulation or exceeds the permitted use, you will need to obtain permission directly from the copyright holder. To view a copy of this license, visit http://creativecommons.org/ licenses/by/4.0/

(C) The Author(s) 2020 